\title{
Tumors escape immunosurveillance by overexpressing the proteasome activator REG $\gamma$
}

Mathilde Boulpicante ${ }^{1}$, Romain Darrigrand ${ }^{1}$, Alison Pierson ${ }^{1}$, Valerie Salgues ${ }^{1}$, Benoit Gaudineau ${ }^{2}$, Mehdi Khaled ${ }^{2}$, Angela Cattaneo ${ }^{3}$, Angela Bachi ${ }^{3}$, Paolo Cascio $^{4}$ and Sébastien Apcher ${ }^{1 *}$

${ }^{1}$ Gustave Roussy, Université Paris Sud, Université Paris Saclay, Inserm U1015, département d'immunologie, Gustave Roussy, 114 rue Edouard Vaillant, 94805 Villejuif, France.

${ }^{2}$ Gustave Roussy, Université Paris Sud, Université Paris Saclay, Inserm U1186, département d'immunologie, Gustave Roussy, 114 rue Edouard Vaillant, 94805 Villejuif, France.

${ }^{3}$ IFOM, the FIRC Institute of Molecular Oncology, via Adamello 16, 20139 Milano, Italy.

${ }^{4}$ Department of Veterinary Sciences, University of Turin, Largo P. Braccini 2, 10095, Grugliasco, Turin, Italy.

* Corresponding Author: Sébastien Apcher, Gustave Roussy, University Paris Sud, University Paris Saclay, INSERM U1015, 114 rue Edouard Vaillant,94805 Villejuif, France. Phone +(33)1 421155 59, E-mail: sebastien.apcher@gustaveroussy.fr

"The authors declare no potential conflicts of interest."

Running title: Up-regulation of REG $\gamma$ in cancer inhibits tumor immune responses 


\begin{abstract}
The success of CD8+ T cell based cancer immunotherapy emphasizes the importance of understanding the mechanisms of generation of MHC-I peptide ligands and possible pathways of tumor cell escape from immunosurveillance. Recently, we showed that peptides generated in the nucleus during the pioneer round of mRNA translation (pioneer translation products, or PTPs) can be a potentially important source of tumor specific peptides, given the presence of aberrant splicing and transcription associated with oncogenesis. Here we show that cancer cells up-regulation of the REG $\gamma$ proteasome regulator results in increased destruction of PTP-derived peptides in the nucleus thus subverting immunosurveillance. These findings add to understanding of the role of REG $\gamma$ in antigen processing and identify it as a druggable target for improving the efficacy of cancer immunotherapy.
\end{abstract}

\title{
Significance
}

With the clear success of CD8+ T cell based immunotherapy, it is critical to understand i) how tumor cells generate MHC-I peptide antigens? and ii) the various mechanisms used by cancer cells to evade immunosurveillance. One of them is to up-regulate the REG $\gamma$ proteasome regulator which results in an increase destruction of MHC-I peptides in the nucleus thus subverting immunosurveillance. 


\section{Introduction}

Cellular immune responses against cancer cells expressing non-self epitopes require the activation of CD8+ T cells by professional antigen presenting cells (pAPCs), which take up external peptide material and present it on their major histocompatibility complex class I (MHC-I) molecules as part of a process called cross-presentation (1). The MHC-I direct and cross-presentation pathways are fundamental processes for the detection and elimination of cells that pose a threat to the host. In recent years, it has been suggested that peptides that are directly presented to the MHC-I restricted pathway are not derived from the degradation of full-length proteins but from so-called defective ribosomal products, or DRiPs (2). In addition, some MHC-I-bound peptides are generated by cryptic translation, which refers to polypeptides synthesized in the cell via non-conventional translational mechanisms. These are either peptides encoded by introns, intron/exon junctions, 5' and 3' untranslated regions, alternate translational reading frames, or even fusion peptides generated by the proteasome (3-6). These observations have led to a shift in focus towards the notion that the degradation of full-length proteins is not the only critical process for antigen production. More recently, we have shown that the antigen presentation is equivalent whether the peptide is expressed from an intron or from an exon is supported by so-called pioneer translation products (PTPs) (7), which are produced by a translation event distinct from canonical translation prior to mRNA splicing. The PTP model is appealing because it offers an explanation of how the immune system "tolerates" tissue-dependent alternative splicing products. By first examining how direct presentation applies to viral mechanisms of immune evasion, we recently demonstrated that PTPs are also a source of peptides for the exogenous MHC-I pathway. Indeed, PTP-derived peptides are cross-presented by pAPCs in order to specifically activate naïve CD8+T cells (8). Moreover, PTPs are present in exosomes that are engulfed by bone marrow dendritic cells (BMDCs) for cross-presentation. Finally, PTPs purified from tumor cells (tumor-associated PTPs or TA-PTPs) have been used in combination with exosomes as a potent immune cancer vaccine (8).

Polypeptides such as PTPs that enter either the endogenous or the exogenous MHC-I pathway must be processed to fit the MHC-I molecule binding groove to elicit an immune response. A key player of this process is the proteolytic system of the eukaryotic cell, the ubiquitin-proteasome system (9). Central to this system is the proteasome, a multicatalytic complex consisting of a $20 \mathrm{~S}$ proteolytic 
core controlled by regulatory complexes that bind to it $(10,11)$. One of these regulatory complexes is the 19S particle, which, along with the 20S proteolytic core, forms the $26 \mathrm{~S}$ proteasome that degrades ubiquitylated and some non-ubiquitylated proteins in an ATP-dependent manner. Other regulatory complexes, including the REG/PA28 family, have been shown to associate with the $20 \mathrm{~S}$ or with asymmetric (i.e. single 19S-capped) $26 \mathrm{~S}$ proteasomes $(12,13)$. The REG family consists of three related subunits, which together form two proteasome regulatory complexes: (i) $R E G \alpha / \beta$, a heteroheptamer formed by REG $\alpha$ and REG $\beta$ subunits, located primarily in the cytoplasm; and (ii) REG $\gamma$, a homoheptamer formed by the REG $\gamma$ subunit, located in the nucleus (14-16). The exact functions and mechanisms of action of REG $\gamma$ remain elusive, as only a limited number of proteins whose degradation is mediated or controlled by this regulator have been described. Among them are cell cycle regulators, including the cyclin-dependent kinase inhibitors p21 and p16, the oncogene SRC-3 and the tumor suppressor p53 (17-20). This paradigm aligns with the proliferation-promoting and anti-apoptotic properties of REG $\gamma$ deduced from an analysis of $\mathrm{KO}$ mice and the observation that $\mathrm{REG} \gamma$ is overexpressed in many cancers and is often associated with a poor prognosis. Moreover, other observations point to a central role for REG $\gamma$ in intranuclear dynamics through the regulation of (i) nuclear bodies (including nuclear speckles, Cajal and PML bodies) and (ii) nuclear trafficking of splicing factors (21-23). Evolutionary analyses have shown that REG $\alpha$ and REG $\beta$ appeared much later in evolution than REG $\gamma$ and diverged concomitantly with the emergence of MHC (24). REG $\gamma$ is important for responses to genotoxic and oxidative stress as well as the impairment of proteasome function, which is for example the case in neurodegenerative diseases (25). Interestingly, it was shown that cellular REG $\gamma$ is massively recruited to proteasomes after non-toxic treatment with proteasome inhibitors (26). Furthermore, recently, a specific interactor of REG $\gamma$ has been identified, also called PIP30, which positively modulates the interaction of REG $\gamma$ to the $20 \mathrm{~S}$ proteasome complex and alters the selectivity of the REG $\gamma / 20$ S proteasome complex towards different peptides (27). We also recently reported that PTP levels inside the nuclear compartment increased when the proteasomal system was blocked using the highly specific proteasome inhibitor epoxomicin (7). 
Although many studies have demonstrated a strong link between REG $\alpha / \beta$ and antigen production in the immune system (28), no data are currently available suggesting a negative or positive role for REG $\gamma$ in this process, although REG-knock-out MEF cells and REG-deficient animal models have been produced and challenged to assess specific immune responses $(29,30)$. Here, we show i) an inverse correlation between the expression of REG $\gamma$ and the presentation of different MHC-I antigenic peptides, ii) REG $\gamma$ involvement in processing of different PTPs and the negative regulation of CD8+ T cell responses against cancer, iii) a decreased presentation of MHC-I PTP-derived antigens due to the downregulation of REG $\gamma$ and iv) knockout of REG $\gamma$ gene causes tumor growth defect in vivo. These findings describe a mechanism by which REG $\gamma$ negatively influences cancer immune responses in an opposite manner compared to the other members of the REG family. These results potentially serve as a starting point for the development of new chemotherapies aiming at decreasing intracellular levels of REG $\gamma$ thus enhancing the production of tumor-associated antigens and stimulating specific immune responses against cancer.

\section{Materials and methods}

\section{T cell hybridomas, cell culture and transfection}

The SIINFEKL:K $\mathrm{K}^{\mathrm{b}}$-specific (B3Z) and the MBP: $\mathrm{K}^{\mathrm{k}}$-specific (MBP CD8+) T cell reporter hybridomas were described previously $(31,32)$. Human A375, WM3526 and WM3682 melanoma cell lines, HT29 and T84 colon tumor cell lines, A549 adenocarcinoma lung cells, and MRC5 "normal" lung cells were cultivated in medium recommended by ATCC.

Cells were transfected with different quantities of expression plasmids along with $2 \mu \mathrm{L}$ of JetPrime according to the manufacturer's protocol (Ozyme). Each plasmid vector contained a Flag tag.

\section{Drugs}

Cells were treated with different drugs: epoxomicin (Peptides International) was used at $300 \mathrm{nM}$ and cisplastin (Sigma) at 5 and $10 \mathrm{mg} / \mathrm{mL}$. 


\section{T cell assay}

Cancer cells were washed twice in $1 \times$ PBS and co-cultured either with the SL8-specific B3Z T cell hybridoma or MBP-specific T cell hybridoma for 16-20 h. Then, the cells were centrifuged at 1,200 rpm for $5 \mathrm{~min}$. The cells were washed twice with $1 \times$ PBS and lysed for $5 \mathrm{~min}$ at room temperature (RT) in the following buffer: $0.2 \%$ TritonX-100, $0.5 \mathrm{M} \mathrm{K}_{2} \mathrm{HPO}_{4}, 0.5 \mathrm{M} \mathrm{KH}_{2} \mathrm{PO}_{4}$. The lysates were centrifuged at 3,000 rpm for $10 \mathrm{~min}$ to pellet cell debris. Next, $45 \mu \mathrm{L}$ of the supernatant was transferred into an optiplate (Packard Bioscience), and a revelation buffer containing $0.01 \%$ methylumbelliferyl $\beta$-D-galactopyranoside (MUG) was then added. The plate was incubated for $3 \mathrm{~h}$ at RT. Human cell lines were transfected with the $\mathrm{Kb}$ expression vector. Both $\mathrm{CD} 8+\mathrm{T}$ cell hybridomas express LacZ in response to the activation of T cell receptors specific for the SIINFEKL peptide (Ovaimmunodominant peptide) in the context of $\mathrm{H} 2-\mathrm{K}^{\mathrm{b}}$ MHC class I molecules or the MBP peptide (myeline basic protein-immunodominant peptide) in the context of $\mathrm{H} 2-\mathrm{K}^{\mathrm{k}} \mathrm{MHC}$ class I molecules. The activity of $\beta$-galactosidase (luminescence) was measured with FLUOstar OPTIMA (BMG LABTECH Gmbh).

\section{CRISPR/Cas9 transfection and selection}

After the transfection of $1 \mu \mathrm{g}$ of CRISPR plasmid vector, cells were sorted 2 days later $(1 \mathrm{cell} /$ well $)$. PCR and Western blotting were performed using the clones; selected clones were sent for sequencing. TOPO TA cloning (Life Technologies) was carried out for selected clones. The CRISPR/Cas9 system was applied to the A375 cell line, and A375 clone number 11 was generated and designated A375c.XI.

\section{FACS Analysis for $\mathrm{H} 2-\mathrm{K}^{\mathrm{b}}$ expression and recovery at the cell surface}

To study the kinetics of endogenous surface $\mathrm{K}^{\mathrm{b}}$ recovery cells were treated with ice-cold citric acid buffer $\left(0.13 \mathrm{M}\right.$ citric acid, $\left.0.061 \mathrm{M} \mathrm{Na}_{2} \mathrm{HPO}_{4}, 0.15 \mathrm{M} \mathrm{NaCl}[\mathrm{pH} 3]\right)$ at $1 \times 10^{7}$ cells per milliliter for $120 \mathrm{~s}$, washed three times with PBS, and resuspended in culture medium. At the indicated time point, an aliquot of cells (generally $1.5 \times 10^{6}$ ) was removed and stained with anti-mouse $\mathrm{H}-2 \mathrm{~K}^{\mathrm{b}}$ PE. All flow cytometry experiments were conducted using the BD LSRII flow cytometer (BD Biosciences) and data are analyzed with the FlowJow software (V10). 


\section{In vitro peptide degradation}

Reconstitution of REG-20S complexes and the degradation of short fluorogenic substrates, the MP-45 and the KH-52 polypeptides (containing the SIINFEKL epitope in their middle) or the MP-46 and the KH-53 polypeptides (containing the MBP epitope in their middle), were performed according to previously described methods (33-35). Briefly, REG $\gamma$ - and REG $\alpha / \beta-20 \mathrm{~S}$ proteasomes were reconstituted by preincubating human 20S constitutive particles (BostonBiochem, USA) with a 6-fold molar excess of REG $\gamma$ (BostonBiochem, USA) or REG $\alpha \beta(34)$ at $37^{\circ} \mathrm{C}$ for $30 \mathrm{~min}$ in $20 \mathrm{mM} \mathrm{HEPES}$, $\mathrm{pH} 7.6$, and $2 \mathrm{mM} \mathrm{NaCl}$ and were immediately used for degradation experiments. For kinetic analysis and to generate peptide products for MS/MS studies, all polypeptides $(50 \mu \mathrm{M})$ were incubated with 20S, REG $\gamma$-20S or REG $\alpha / \beta-20 \mathrm{~S}(20 \mathrm{nM})$ for $8 \mathrm{~h}$ at $37^{\circ} \mathrm{C}$ in $20 \mathrm{mM}$ HEPES, $\mathrm{pH} 7.6,2 \mathrm{mM} \mathrm{NaCl}$. To assay the peptides generated during protein degradation, we measured the appearance of new amino groups using fluorescamine as previously described (35). In brief, at the end of the incubation, the peptide products were separated from undegraded polypeptides by ultrafiltration through a membrane with a 3-kDa cutoff (Nanosep, Pall, USA), and these samples were assessed with fluorescamine (Sigma) and used for MS/MS analysis.

\section{Liquid chromatography-tandem MS (LC-MS/MS) analysis}

Five-microliter samples from the $20 \mathrm{~S}-/+$ REG $\gamma$ experiments containing approximately 130 pmol $\mathrm{NH}_{2} / \mu 1$ were loaded onto StageTips $\mu \mathrm{C} 18$ (36,37); peptides were eluted in $40 \mu 1$ of $80 \%$ acetonitrile in $0.1 \%$ formic acid. The acetonitrile was allowed to evaporate in a Speed-Vac, and then the samples were resuspended in $6 \mu 1$ of eluent A (see the composition below) for nLC-MS/MS analysis. Two microliters of each sample was injected as technical replicates into a nLC-ESI-MS/MS quadrupole Orbitrap QExactive-HF mass spectrometer (Thermo Fisher Scientific). Peptide separation was achieved using a linear gradient from $95 \%$ solvent $\mathrm{A}$ ( $2 \% \mathrm{ACN}, 0.1 \%$ formic acid) to $50 \%$ solvent $\mathrm{B}$ (80\% acetonitrile, $0.1 \%$ formic acid) for $23 \mathrm{~min}$ and from 60 to $100 \%$ solvent B for 2 min at a constant flow rate of $0.25 \mu 1 / \mathrm{min}$ on a UHPLC Easy-nLC 1000 (Thermo Scientific) connected to a $25-\mathrm{cm}$ fused-silica emitter with an inner diameter of $75 \mu \mathrm{m}$ (New Objective, Inc. Woburn, MA, USA), packed in-house with ReproSil-Pur C18-AQ 1.9- $\mu \mathrm{m}$ beads (Dr. Maisch Gmbh, Ammerbuch, 
Germany) using a high-pressure bomb loader (Proxeon, Odense, Denmark). MS data were acquired using a data-dependent top-15 method for HCD fragmentation. Survey full-scan MS spectra (300$1750 \mathrm{Th}$ ) were acquired in the Orbitrap at a resolution of 60,000 , an AGC target of $1^{\mathrm{e} 6}$, and an IT of $120 \mathrm{~ms}$. For the HCD spectra, the resolution was set to 15,000 at $\mathrm{m} / \mathrm{z}, 200$, with an AGC target of $1^{\mathrm{e} 5}$, an IT of $120 \mathrm{~ms}$, an NCE of $28 \%$ and an isolation width of $3.0 \mathrm{~m} / \mathrm{z}$.

\section{Data processing and analysis}

For quantitative proteomic analysis, raw data were processed with MaxQuant (ver. 1.5.2.8) and searched against a database containing only the sequence of the KH-52 intron SIINFEKL. No enzyme specificity was selected, and there were no differences between I and L. The mass deviation for MSMS peaks was set at $20 \mathrm{ppm}$, and the peptide false discovery rate (FDR) was set at 0.01 ; the minimal length required for a peptide identification was eight amino acids. The list of identified peptides was filtered to eliminate reverse hits. Statistical analyses were performed with Perseus (ver. 1.5.1.6) considering the peptide intensity; normalization based on the Z-score and imputation was applied. Significant peptides were determined with a t-test, Benjamini Hochberg correction and FDR $<0.05$ (more stringent assignments), or a t-test with a p-value of 0.05 (less stringent assignments). Only significant peptides were used for supervised hierarchical clustering analysis.

\section{Tumor challenge in-vivo}

C57B1/6J female mice were obtained from Harlan Laboratories. NU/NU nude mice were obtained from Charles River. 7 week-old mice were injected subcutaneously into the right flank with $1 \times 10^{5}$ MCA205 wild type (WT) cells or 1x105 Cas9-REG? MCA205 clones. Area of the tumor was recorded every 3 to 4 days until ethical limit points are reached. All animal experiments were carried out in compliance with French and European laws and regulations.

\section{RESULTS}

Inverse correlation between the expression of REG $\gamma$ and antigen presentation in multiple cancer cell lines.

A series of studies have reported the overexpression of REG $\gamma$ in different cancer types (3840). To clarify the specific role of REG $\gamma$ in MHC-I antigen presentation, we selected different cancer 
cell lines and examined REG $\gamma$ mRNA and protein expression levels. First, we analyzed REG $\gamma$ mRNA levels by real-time qRT-PCR in three human melanoma cell lines (A375, WM3526 and WM3682), one human lung cancer cell line (A549) and two colon cancer cell lines (HT29 and T84), and we observed that REG $\gamma$ was notably upregulated in each cell line compared to the control normal lung cell line MRC5 (Fig. 1A). Next, we measured REG $\gamma$ protein expression and observed that it was overexpressed in all cancer cell lines compared with MRC5 normal cells, although levels of REG $\gamma$ overexpression varied between the different cell lines analyzed. Indeed, REG $\gamma$ was highly overexpressed in melanoma and colon cancer cell lines, expressed at low levels in the lung cancer cell line A549, and demonstrated very low expression in the normal cell line (Fig. 1B). Furthermore, we assessed the cellular distribution of REG $\gamma$ in these cancer cell lines. Figure 1C shows that endogenous REG $\gamma$ was localized in the nucleus of all cell lines analyzed independently of its expression levels.

To evaluate whether the observed differences in REG $\gamma$ expressions in cancer and normal cell lines contribute to changes of antigen production and presentation, we examined the presentation of PTP-derived-antigens at the cell surface of these cell lines. To achieve this goal, we expressed the mouse MHC-I K ${ }^{\mathrm{b}}$ molecule and Glob-intron-SL8 constructs in these human cell lines, which enabled us to examine the production of specific SL8-comprising-PTPs as described previously $(6,7)$. Using a SIINFEKL:K $\mathrm{K}^{\mathrm{b}}$ (B3Z) T cell hybridoma (31) that specifically detects the SL8 epitope presented on $\mathrm{K}^{\mathrm{b}}$ molecules, we first found that the antigen presentation was lower in all cancer cell lines tested than in the normal MRC5 cell line (Fig. 1D, left panel). Interestingly, we observed a complete inverse correlation between PTP-derived-SL8 presentation and REG $\gamma$ expression in all cell lines tested. This result suggested that REG $\gamma$ overexpression negatively affected PTP-derived antigen presentation (compare Figs. 1B and 1D). Crucially, this result was not merely due to differences in the cellular level of the $\beta$-globin construct, which in fact were higher in tumor cell lines overexpressing REG $\gamma$ (Supplementary Fig. 1A). Moreover, FACS analysis showed that overall H2- $\mathrm{K}^{\mathrm{b}}$ expression differed among the cancer cell lines tested, but it did not correlate with the expression of SL8 as determined with the B3Z assay. In fact, we observed that MHC-I molecules were more abundant at the cell surface 
of tumor cell lines overexpressing REG $\gamma$ and exhibiting a decrease in PTP-dependent antigen presentation (Supplementary Fig. 1B).

In order to rule out the possibility that the negative role of REG $\gamma$ on PTP dependent antigen presentation could be restricted to the SL8 epitope or the $\mathrm{K}^{\mathrm{b}}$ molecule, we determined whether also the presentation of the $\operatorname{MBP}(79-87)$ epitope, which is derived from the Myelin Basic Protein (MBP) and is presented on $\mathrm{K}^{\mathrm{k}}$ molecules was affected by the overexpression of REG $\gamma$ in different cancer cell lines . Using the specific MBP CD8+ T cell hybridoma (32) we could obtain results similar to those previously observed with the PTP-derived-SL8, that is to say a complete inverse correlation between PTP-derived-MBP presentation and REG $\gamma$ expression in all cancer cell lines tested (Fig. 1D, right panel). All together, these results indicate: i) a negative role of REG $\gamma$ in the MHC-I antigen presentation pathway since in all cell lines overexpressing it the $\beta$-globin protein and the MHC-I molecules were more abundant while the antigen presentation was reduced and ii) that the inverse correlation between REG $\gamma$ expression and antigen presentation is not restricted to a specific epitope or MHC-I molecule.

We then wanted to determine whether the previously observed differences in SL8 antigen presentation were restricted to intron-derived tumor-associated antigens or if they were also observed with an exon-derived tumor-associated antigen. To this purpose, we analysed cells expressing mouse MHC-I K $\mathrm{K}^{\mathrm{b}}$ molecule and Glob-exon-SL8 construct or ovalbumin cDNA, in which the SL8 epitope is found in its correct setting. As shown in Figure 1E, antigen presentation showed an inverse correlation with REG $\gamma$ expression in all cell lines tested, independently of the position of the antigenic epitope in the exon sequence (left panel) or in cDNA constructs (right panel).

Furthermore, we expressed in the different human cell lines the mouse MHC-I H2-K molecule and the SL8-minigene construct, which contains only the presented 8-amino-acid sequence of SL8, and observed that differences in REG $\gamma$ expression did not correlate with SL8 antigen presentation (Supplementary Fig. 1C). Hence, the effects of REG $\gamma$ on MHC-I antigen presentation only occurred with longer polypeptides containing the presented MHC-I antigenic peptides, indicating that REG $\gamma$ directly acts on epitopes processing and not on subsequent steps of class-I presentation. 
All together these results clearly support the hypothesis that the proteasome regulator REG $\gamma$ is involved in the inhibition of MHC-I presentation of antigens present in exon as well as in intronderived PTPs, a mechanism that may allow tumor cells to avoid immune responses against conventional and unconventional epitopes.

\section{Exogenous overexpression of REG $\gamma$ decreases MHC-I antigen presentation.}

In the rest of our study we decide to use the Glob-intron SL8 construct since, due to its nuclear localization it is the most appropriate tool to investigate the role of a nuclear proteasomal regulator on PTPs dependent antigen presentation. To study the specific effects of REG $\gamma$ on PTP-dependent MHC-I antigen presentation, MRC5 lung fibroblast cells and A549 lung cancer cells, which express REG $\gamma$ at very low levels, (see Figs. 1A and 1B), were therefore co-transfected with the mouse MHC-I K molecule, the Glob-intron-SL8 and an exogenous Flag-REG $\gamma$ construct. Using, Western blotting (Fig. 2A), immunofluorescence (Fig. 2B) or qRT-PCR (Supplementary Figure 2), we monitored the expression of the exogenous REG $\gamma$ construct and compared it to the expression of endogenous REG $\gamma$. Notably, the overexpressed exogenous REG $\gamma$ showed the same expression pattern and localization as the endogenous REG $\gamma$. Next, we examined the antigen presentation of the SL8 epitope in these cell lines. The introduction of increasing amounts of REG $\gamma$ in both A549 and MRC5 cell lines, which express fixed amounts of the Glob-intron-SL8 and MHC-I K ${ }^{\mathrm{b}}$ molecules, resulted in a dose-dependent decrease in the activation of the B3Z hybridoma (Fig. 2C). This observation supports the hypothesis that the overexpression of REG $\gamma$ has a clear-cut negative effect on PTP-dependent antigen presentation.

As mentioned earlier, REG $\gamma$ is not the sole regulator of the proteasomal pathway; 20S proteasome also binds to the REG $\alpha / \beta$ regulator, which has been shown to be implicated in the accurate processing of MHC-I antigenic epitopes (14). To investigate more in detail at which exact level of the proteasomal pathway REG $\gamma$ contributes to PTP-dependent antigen presentation regulation, we determined whether the overexpression of REG $\gamma$ influenced the expression of endogenous REG $\alpha$. Figure 2D shows that increasing the amount of exogenous REG $\gamma$ did not affect the expression of REG $\alpha$. This result therefore demonstrates that the decrease in PTP-dependent antigen presentation 
causes by REGg in different cancer cell lines is not due to a decrease in the expression of the regulator REG $\alpha$ but more likely it is the result of a direct effect on the 20S proteasome. In fact, the presentation of antigenic peptides depends on proteolytic processing by the $20 \mathrm{~S}$ proteasome complex. To ensure that differences in the production of SL8 peptides by the proteasome are unambiguously attributable to the overexpression of REG $\gamma$, we treated MRC5 cells expressing increasing amounts of REG $\gamma$ and a fixed amount of Glob-intron-SL8 and MHC-I K ${ }^{\mathrm{b}}$ molecules with the 20S proteasome-specific inhibitor epoxomicin and examined SL8 presentation. Interestingly, while non-treated cells displayed a decrease in antigen presentation with REG $\gamma$ overexpression, a partial increase in MHC-I antigen presentation was observed when cells were treated with epoxomicin (Fig. 2E). This observation supports the idea that REG $\gamma$ does not exert unspecific processing effects on MHC class I antigen presentation in the overexpressing cell lines but instead confers a real and specific effect on the $20 \mathrm{~S}$ core proteasome. Moreover, as a control for the real efficacy of the proteasomal inhibitor under the condition used in the treated cell lines, we examined p21 protein levels, which has been reported to be regulated specifically by the REG $\gamma$-20S proteasome complex in an ubiquitin and ATP-independent process (18). p21 protein levels increased following $18 \mathrm{~h}$ of epoxomicin treatment, even though the treated cells overexpressed the exogenous REG $\gamma$ regulator (Fig. 2F).

Therefore, all these results confirm that the overexpression of REG $\gamma$ does not result in (i) a decrease in MHC-I expression or export, or (ii) a decrease in the expression of the other members of the REG family, but does directly affect proteasomal proteolytic activities.

\section{Knockdown and knock-out of the regulator REG $\gamma$ promote antigen presentation.}

Considering our finding showing that endogenous overexpression of the REG $\gamma$ regulator negatively affects MHC-I antigen presentation in cancer cells, we speculated whether REG $\gamma$ knockdown might restore PTP-dependent antigen presentation. To test this hypothesis, we silenced REG $\gamma$ by transient siRNA treatment in human melanoma and colon cancer cell lines. As expected, REG $\gamma$ knockdown (Fig. 3A) enhanced p21 protein levels in all cancer cell lines compared to scrambled siRNA (Fig. 3B). More importantly in terms of PTP-dependent antigen presentation, we 
observed a close correlation between REG $\gamma$ knockdown and the increase of the SL8 epitope at the cell surface (Fig. 3C).

The expression of REG $\gamma$ is controlled by several mechanisms inside the cell. miRNA-7, which has been reported as a tumor suppressor $(41,42)$ and is downregulated $(43,44)$ in several human cancers, also negatively controls the expression of the regulator REG $\gamma$ in lung cancer (45). The A375 melanoma cancer cell line, which in our hands expressed the highest levels of REG $\gamma$, was therefore transfected with increasing amounts of miRNA-7. Of great interest, we observed that miRNA-7 inhibited REG $\gamma$ expression (Supplementary Fig. 3A) and led to an increase in PTP-SL8-dependent antigen presentation (Supplementary Fig. 3B) $(41,42)(41,42)(41,42)(41,42)(41,42)(40,41)(38,39)$.

In addition, treatment of cancer cells with the chemotherapeutic molecule cisplatin has been shown to induce a decrease in mRNA and protein levels of REG $\gamma$ (46) (and Supplementary Fig. 3C). Accordingly, treatment of A375 cells with cisplatin restores the expression of PTP-dependent antigens at the cell surface (Supplementary Fig. 3D). All together, these results support the idea that REG $\gamma$ downregulation restores PTP-derived antigen presentation and indicate a possible chemotherapeutic approach to achieve this goal. To clarify ore in detail the functional importance of REG $\gamma$ in cancer immune escape, we used the CRISPR/Cas9 system to create a melanoma Cas9-A375 REG $\gamma$-knock-out cell line (A375cXI). The heterozygous knock-out A375cXI was confirmed by Sanger sequencing, qRT-PCR and Western blot analysis. The A375cXI cell line did not express REG $\gamma$ mRNA (Fig. 3D) or its corresponding protein (Fig. 3E). As a control for the loss of REG $\gamma$ expression, the p21 protein accumulates in this cell line, as in cells that were knocked down with specific REG $\gamma$ siRNAs compare to the parental A375 WT line (Fig. 3E). Crucially, when analyzed for antigen presentation and processing, A375cXI cells proved able to strongly activate the PTP-dependent immune response in contrast to A375 WT (Fig.3F). Moreover, the level of antigen presentation in these cell lines was even higher than the control MRC5 line, which weakly expresses REG $\gamma$ (Fig. 3F). Differences in REG $\gamma$ levels are likely to account for this result, since both the tested cell lines (A375cXI and A375 WT) expressed equal amounts of exogenous Glob-intron-SL8 protein (Fig. 3G, top panel) as well as equal amounts of the mouse MHC class I molecule at their cell surface (Fig. 3G, bottom panel). 
All together these results clearly confirm that there is an inverse correlation between the expression of REG $\gamma$ and PTP-dependent antigen presentation in cancer cell lines. This effect is likely due to a close control of the proteasomal degradative pathway and not to an overall effect of REG $\gamma$ on MHC-I molecules expression and export to the cell surface.

\section{REG $\gamma$ regulates the nuclear proteasomal pathway.}

In our previous studies, we demonstrated that PTPs are produced by a translation event that is distinct from canonical translation and occurs prior to mRNA splicing, supporting the idea that PTPs are generated by a nuclear translation event. Furthermore, we have also reported that treatment with the proteasome inhibitor epoxomicin increases the amount of PTPs within the nuclear compartment, indicating that the nuclear proteasome may be involved in the processing and/or degradation of PTPantigenic epitopes (7). Based on these studies and all our results so far, we next sought to understand how REG $\gamma$ contributes to the specific inhibition of PTP-dependent cancer immune responses and in which cell compartment this inhibition occurs. Antigenic epitopes derived from PTP processing are generated either by a cytosolic or nuclear proteasomal complex, in which the REG family plays an essential role. As proteasomal regulator, REG $\gamma$ has to bind to the $20 \mathrm{~S}$ proteasome to control protein or polypeptide degradation and this interaction should occur in the nucleus. A REG $\gamma$ gene sequence mutation that leads to the replacement of Asn 151 by Tyr (N151Y) has been reported to impair the ability of the regulator to activate the trypsin-like activity of the $20 \mathrm{~S}$ proteasome (47). A375cXI cells were transfected with increasing amounts of Flag-REG $\gamma$ WT or mutated Flag-REG $\gamma$ N151Y. First, we showed by western-blotting that compared to the WT REG $\gamma$, the mutated regulator was no longer able to degrade the p21 protein likely as a direct consequence of its inability to activate the trypsin-like activity of the $20 \mathrm{~S}$ proteasome. Moreover, the expression of the other members of the REG family was not impacted under all the experimental conditions used (Fig. 4A). More importantly, FlagREG $\gamma \mathrm{N} 151 \mathrm{Y}$ inhibited SL8 antigen production and presentation at the cell surface from Glob-intronSL8 less efficiently than Flag-REG $\gamma$ WT (Fig. 4B). This inhibition of SL8 presentation again was not attributable to a decrease in expression of the other REG family members shown to be involved in antigen presentation. Two explanations are therefore consistent with this result: i) exogenous mutated 
Flag-REGyN151Y may no longer be localized in the nucleus; or ii) exogenous mutated FlagREG $\mathrm{N} 151 \mathrm{Y}$ may have lost its capacity to bind to the 20S proteasome and, therefore, contrary to FlagREG $\gamma \mathrm{WT}$ it is no longer able to inhibit proteasome activity. To discriminate between these two alternative hypotheses, we first examined the distribution of the mutated Flag-REG 7 N151Y as well as its capacity to bind to the $20 \mathrm{~S}$ proteasome in cellulo. As shown in Figure 4C, the mutated and WT exogenous REG $\gamma$ regulators are both localized in the nucleus. Since we did not observe any differences in the localization that could explain the differences in the production of MHC class I epitopes between the cell lines expressing the mutated or the WT regulators, we determined whether mutated Flag-REG $\gamma$ N151Y retained the capacity to bind to the nuclear 20S proteasome in cellulo. To achieve this goal, we used proximal ligation assay (PLA)-labeled secondary antibodies, which allow the detection of two primary antibodies in close proximity. Using a combination of PLA-labeled antiFlag and anti-proteasomal $\alpha 4$ antibodies, we observed a specific signal in the nuclear compartments of cells expressing the mutated and WT REG $\gamma$ constructs, revealing the co-localization of both form of REG $\gamma$ with the $20 \mathrm{~S}$ proteasome (Fig. 4D). Furthermore, as a control, anti-Flag alone under these conditions produced no PLA reaction, and no positive signals were detected in non-transfected cells (Fig. 4D, top panel). We confirmed further the interaction between WT Flag-REG $\gamma$ and mutated FlagREG $\gamma \mathrm{N} 151 \mathrm{Y}$ with the $20 \mathrm{~S}$ core proteasome by coimmunoprecipitation using an antibody directed against the REG $\gamma$ regulator (Fig. 4E). Localization and capacity to bind to the $20 \mathrm{~S}$ core were equivalent between exogenous Flag-REG $\gamma$ WT and mutated Flag- REG $\gamma$ N151Y, again demonstrating that the REG $\gamma$-20S proteasome complex is involved in the processing/degradation of PTP-derived antigenic peptides.

To further define the compartment hosting REG $\gamma$-dependent PTP degradation, we generated an exogenous Flag-REG $\gamma$ construct that did not contain a nuclear localization signal (NLS). Unlike Flag-REG $\gamma$ WT, Flag-REG $\gamma \Delta$ NLS was expressed throughout the cell and was not confined to the nucleus (Fig. 4C). Interestingly, the expression of Flag-REG $\gamma \Delta \mathrm{NLS}$ was not confined to the cytoplasm, suggesting that REG $\gamma$ contains more than one nuclear localization signal. The combination 
of PLA-labeled anti-Flag and anti-proteasomal $\alpha 4$ antibodies produced a specific signal in the cytoplasmic compartments of cells expressing the Flag-REG $\gamma \Delta$ NLS and Flag-REG $\gamma$ WT constructs. We also observed staining corresponding to an interaction between Flag-REG $\gamma \Delta$ NLS and the $20 \mathrm{~S}$ core in the nucleus. This result indicates that Flag-REG $\gamma \Delta$ NLS, which was localized throughout the cell, retained the capacity to bind to the $20 \mathrm{~S}$ proteasome in both the cytoplasm and the nucleus (Fig. 4D). We also confirmed the interaction between Flag-REG $\gamma$ WT and Flag-REG $\gamma \Delta$ NLS with the $20 \mathrm{~S}$ core proteasome by coimmunoprecipitation using an antibody directed against the REG $\gamma$ regulator (Fig. 4E). Moreover, A375 $\Delta$ REG $\gamma$ cells transfected with Flag-REG $\gamma \Delta$ NLS slightly prevented nuclear $20 \mathrm{~S}$ proteasome degradation of the p21 protein in a REG $\gamma$-dependent manner compared to cells transfected with Flag-REG $\gamma$ WT (Fig. 4F). This result suggests that the low levels of Flag-REG $\gamma \Delta N L S$ in the nucleus retained the capacity to partially activate the nuclear $20 \mathrm{~S}$ proteasome and, furthermore, demonstrate that the degradation of the p21 protein is a nuclear and not a cytoplasmic event (Fig. 4F). In parallel, when we examined the effects of the partial relocalization of Flag-REG $\gamma \Delta \mathrm{NLS}$ in the cytoplasm on the antigenic presentation pathway, we observed an increase in SL8-PTP-dependent antigen presentation compared to the loss of antigen presentation in cells expressing exogenous nuclear Flag-REG $\gamma$ WT (Fig. 4G).

All of these results hence demonstrate that REG $\gamma$ is involved in PTP-dependent antigen presentation by binding to the $20 \mathrm{~S}$ proteasome in the nucleus.

\section{REG $\gamma$ promotes the degradation of MHC class I PTP-derived antigenic epitopes.}

The above results raised at least two alternative hypothesis regarding the role of REG $\gamma$ in the PTP-dependent proteasomal degradation pathway: i) REG $\gamma$ may inhibit 20S proteasomal peptidase activities are responsible for producing antigenic peptides with the correct characteristics (i.e. length and anchor residues) to serve in MHC-I pathway, with a consequent decrease in production of PTPdependent epitopes; or ii) REG $\gamma$ may stimulate specific 20S proteasomal activities that cause further degradation of the correct size MHC-I antigenic epitopes. Recently, we showed that inhibition of the proteasomal pathway with epoxomicin leads to increased amounts of unprocessed PTPs in cells and 
promotes the cross-presentation of longer polypeptides compared with untreated cells (8). To test the first hypothesis, we transfected different cancer cell lines, normal MRC5 fibroblasts and the A375 $\triangle$ REG $\gamma$ cell line with the Glob-intron-SL8 construct and incubated them with mouse BMDCs for $24 \mathrm{~h}$. We observed a reduction in B3Z activation when the tumor cell lines were incubated with BMDCs compared to the activation levels observed with A375 $\mathrm{REG} \gamma$ and MRC5 cells (Fig. 5A). The decrease in cross-presentation in the different cell lines overexpressing REG $\gamma$ suggested that REG $\gamma$ did not inhibit PTP processing, leading to an accumulation of unprocessed polypeptides, which would have been a source of material for the exogenous MHC-I pathway.

To confirm that REG $\gamma$ did not inhibit the capacity of $20 \mathrm{~S}$ proteasome to hydrolyze long polypeptides containing epitopes, we assessed the effects of this regulator on the $20 \mathrm{~S}$ core particle cleaving properties by using an in vitro degradation assay. To achieve this goal, a 45-mer (MP-45), and a 52-mer (KH-52) precursor peptide containing the SIINFEKL epitope and a 46-mer (MP-46), and a 53-mer (KH-53) precursor peptide containing the $\operatorname{MBP}(79-87)$ epitope were synthesized (Supplementary Fig. 4). The 45-mer and the 46-mer precursor peptides correspond to the amino acid sequence of the $\beta$-globin exon region in which the SL8 or the MBP(79-87) epitopes are introduced in the construct used in Figure 1E. The 52-mer and the 53-mer precursor peptides correspond to the amino acid sequence of the $\beta$-globin intron region in which the SL8 and the MBP(79-87) epitope are introduced in the same construct used in Figure 1D. Importantly, preliminary studies demonstrated that the commercial enzymes used in these experiments were active and free of contaminant proteases, as shown by the ability of epoxomicin to completely inhibit the chymotrypsin- and trypsin-like activities of these preparations (Supplementary Table 1). KH-52 and KH-53 were then incubated in vitro with human constitutive $20 \mathrm{~S}$ proteasome at $37^{\circ} \mathrm{C}$ for several hours, and the rates of peptide bond hydrolysis were assessed by measuring the generation of new amino groups with fluorescamine (35). Under these conditions, KH-52 and KH-53 were clearly degraded by h20Sc at linear rates over the entire time course of incubation (Fig. 5B). Moreover, the rates of peptide bond hydrolysis were not appreciably enhanced in the presence of REG $\gamma$ and appeared to be only slightly lower than the rates measured when the proteasome associated with REG $\alpha / \beta$ (Fig. 5B, compare blue and red curves, left 
and right panels). Furthermore, indicative of absolute proteasome-dependent degradation, peptide hydrolysis was completely prevented in the presence of $20 \mu \mathrm{M}$ epoxomicin (Fig. 5B, green curve, left and right panels). Similar results were also obtained when MP-45 and MP-46 precursor peptides were incubated under the same experimental conditions (Fig. 5C).

We then assessed the second hypothesis namely that REG $\gamma$ stimulates $20 \mathrm{~S}$ proteasome in a manner that leads to complete degradation of MHC class I epitopes. In fact, although binding of REG $\gamma$ did not result in significantly increased rates of substrate hydrolysis (Fig. 5B and 5C), it may modify proteasome cleavage specificities to generate different patterns of peptide products as already extensively demonstrated for REG $\alpha / \beta$ (34). Such a change in enzymatic properties may potentially affect the formation of specific antigenic peptides such as SIINFEKL. To test directly this possibility, peptides released during KH-52 hydrolysis by equimolar amounts of $20 \mathrm{~S}$ with and without REG $\gamma$ were analyzed by tandem mass spectrometry (MS/MS). Importantly, shorter peptides (representing the great majority of proteasomal products $(34,48,49))$ were excluded to minimize false-positive identification and signals originating from small chemical compounds and therefore were not identified or quantified. In contrast, peptides with the correct size to bind to MHC-I heterodimers (i.e., 8-10 mers) were accurately analyzed. Using this approach, we identified 76 different peptides from KH-52, ranging in length from 8 to 16 residues and derived from the entire sequence of the substrate. Importantly, some peptides were generated exclusively by one form of the proteasome (i.e., 20S or REG $\gamma$-20S) (supplementary Table 2), while several others were released by both proteasomal forms. Although MS/MS does not provide quantitative information regarding the absolute abundance of the peptides detected, it is possible to assess their relative amounts by comparing the corresponding ion intensities measured in sequential MS/MS analyses. Therefore, we used ion intensities to quantify the relative amounts of single fragments generated from KH-52 in both degradation reactions. Remarkably, this analysis demonstrated that different amounts of peptide products are released by 20Sc and REG $\gamma$-20S (Fig. 5D). Most importantly, we demonstrated that the generation of SIINFEKL was strikingly suppressed in the presence of REG $\gamma$, as inferred by a two-log value reduction in its ion intensity (Fig. 5E). 
All of these results unambiguously demonstrate that the regulator REG $\gamma$ has the capacity to modify the cleavage properties of the $20 \mathrm{~S}$ proteasome in the nuclear compartment in such a way that PTP-derived MHC class I antigenic peptides are destroyed rather than correctly processed and released.

\section{Knockout of REG $\gamma$ gene causes tumor growth defect.}

Considering the previous results obtained with the human cell lines on the expression of REG $\gamma$, we decided to look at the REG $\gamma$ mRNA level and REG $\gamma$ expression in the mouse sarcoma MCA205 and mouse melanoma B16F10 cell lines and to compare them with those observed in the primary skin fibroblast cell line B6. We observed by qRT-PCR that REG $\gamma$ mRNA was notably upregulated in MCA205 tumor cell line compared to the B6 cell line (Supplementary Fig. 5A). Furthermore, we noticed an overexpression at the protein level of REG $\gamma$ in MCA205 murine cancer cell line compared to B6 cell line (Fig. 6A). To evaluate whether the extent of the difference in REG $\gamma$ expression in murine cell lines contributes to antigen production and presentation, murine sarcoma and primary skin fibroblast cell lines were transiently induced to express the Globin-SL8-intron construct and were compared in terms of B3Z hybridoma activation. We observed that antigen presentation in sarcoma cancer cell line tested was lower than that in the fibroblast cell line supporting our previous results with the human cancer cell lines (Fig. 6A). To further depict the role of REG $\gamma$ in tumorigenicity and cancer immune response, we genetically deleted REG $\gamma$ in the MCA205 sarcoma cancer cell line (Fig. 6B, upper panel and Supplementary Fig. 5B). Then Cas9-REG $\gamma$ MCA205 and MCA205 cells were transiently expressing the Globin-SL8-intron construct and were compared in terms of B3Z hybridoma activation. In this way we observed that Cas9-REG $\gamma$ MCA205 clones induce a stronger activation of $\mathrm{B} 3 \mathrm{Z}$ compared to the parental murine cell line (Fig. 6B, lower panel). In the same manner, and to support our above results showing that downregulation of REG $\gamma$ induce a better cancer immune response due to the non-degradation of PTP-dependent antigens, rather than as a consequence of an overall increased expression of MHC-I molecules at the cell surface, we acid stripped cell surface class I molecules and measured the recovery of surface class I expression. The Cas9-REG $\gamma$ MCA205 clone does not show any increase in MHC-I K ${ }^{\mathrm{b}}$ molecules at the cell surface compare to the 
WT cancer cell line. Rather, a decrease in the amount of total MHC- I molecules is observed although these cells elicit a better cancer immune response compared to that obtained in presence of REG $\gamma$ overexpression (Supplementary Fig. 5C). This result convincingly supports the idea that the effect of REG $\gamma$ on antigen presentation takes place earlier than the loading of the antigenic peptides and the exporting steps of the MHC class I peptide complex.

The proteasome regulator REG $\gamma$ has been reported to have a specific effect on tumorigenicity. In fact it has been shown that REG $\gamma$ contributes to the proliferation and metastasis of different cancer types. To gauge the effect of REG $\gamma$ on cancer immune response and tumorigenicity in vivo, mice were subcutaneously injected into the flank with MCA205 WT cells or with several Cas9-REG $\gamma$ MCA205 clones respectively. As shown in figure 6C, the Cas9-REG $\gamma$ MCA205 clones originate smaller tumors than the parental cell line at day 20 after challenge. This result indicates that the loss of REG $\gamma$ induces a decrease in the tumorigenicity of sarcoma cells. To validate that this decrease in tumorigenicity was due at least in part to a specific activation of the cancer immune response, as we observed in vitro, Nude/Nude mice were subcutaneously injected with MCA205 cells or several Cas9-REG $\gamma$ MCA205 clones. As shown in figure 6D, the relative growth of the different Cas9-REG $\gamma$ MCA205 clones in nude mice was higher than that of the WT cells. This difference in tumor growth between the Cas9REG $\gamma$ MCA205 clones and the parental cell line in immunodeficient nude mice demonstrates the crucial role of REG $\gamma$ in tumorigenicity but also in antigen presentation and tumor immune responses.

These in vivo results confirm all our previous in vitro finding clearly demonstrating that there is an inverse correlation between REG $\gamma$ expression and antigen presentation in cancer and showing that cancer cells overexpress REG $\gamma$ to destroy PTP-dependent antigenic peptides in order to reduce specific tumor immune responses.

\section{Discussion}

This study describes for a first time a distinct and unexpected role of the REG $\gamma$-proteasome complex, which proved to be responsible for MHC-I antigens degradation in cancer. Specifically, we demonstrate an inverse correlation between REG $\gamma$ expression and MHC class I antigen presentation in 
cancers, thus revealing a new function of this regulator in blunting immuno-responses by modulating the activity of the proteasome processing pathway in vitro and in vivo.

Contrary to our results, approximately twenty years ago, REG $\gamma-/$ - mice were reported to exhibit neither positive nor negative effects in terms of antigen presentation. In fact, Barton et al. demonstrated that REG $\gamma-/$ - mice did not show notable impairment in antigen presentation after viral infection (29). The only observed phenotypes reported so far for REG $\gamma-/$ - mice so far are a small reduction in the numbers of specific CD8+ T lymphocytes and growth retardation $(29,30)$. In this study, we clearly demonstrate for the first time a specific role for REG $\gamma$ in MHC-I antigen processing and presentation. First of all, we show that REG $\gamma$ acts as a negative regulator of MHC-I antigen presentation in cancer by destroying in vitro and in cellulo MHC-I peptides generated during proteasomal degradation of PTPs. Furthermore, we demonstrate that overexpression of REG $\gamma$ negatively affects cancer immune response in vivo. Considering our results, it is therefore not surprising that Barton et al. did not observe any effects on antigen presentation in mice lacking REG $\gamma$. In fact, we observed a defect in MHC-I antigen presentation in cancer cell lines or in mice only when REG $\gamma$ was naturally overexpressed. Indeed, when REG $\gamma$ was knocked down in different cancer cell lines using either siRNA, miRNA-7 overexpression, cisplatin treatment, or CRISPR technology, we observed an important strengthening of CD8 $+\mathrm{T}$ cell activation and an enhanced immunological response against tumor progression in vivo. In contrast, in normal cell lines in which REG $\gamma$ is not overexpressed or when we rescue the expression of REG $\gamma$ in the A375 melanoma CRISPR cell line (A375cXI), we have been capable to inhibit MHC-I antigen presentation, at the same level observed in cancer cell lines naturally overexpressing REG $\gamma$. It is generally believed that only $R E G \alpha / \beta$ in association with the immunoproteasome is able to affect the production of MHC-I epitopes. Strikingly, our results clearly demonstrate a close correlation between the expression of REG $\gamma$ and tumor immune evasion. Thus, not only the heteroheptamer $\mathrm{REG} \alpha / \beta$ plays a role in antigen presentation but REG $\gamma$ does as well, although exerting an opposite effect. In fact, we proved that knocking out of REG $\gamma$ reduces the growth of sarcoma MCA205 in immunocompetent mice. However, when we performed the same experiment in Nude nu/nu mice, which are deficient in $\mathrm{T}$ cells but not in B or innate immune 
cells, we did observe an increased tumor growth of several Cas9-REG $\gamma$ MCA205 clones compared to the MCA205 WT cells that overexpress REG $\gamma$. This crucial experiment firmly established that knocking out REG $\gamma$ induces the presentation of new PTP-MIPs that are recognized by CD8+ T cells, and so delaying the tumor growth. In fact, the tumor growth delay is likely to be a consequence of the escape from proteasomal destruction of new PTP-MIPs arising from translation of pre-spliced mRNAs leading to the apparition of epitopes derived from non-coding regions.

As mentioned above, many studies have reported that REG $\gamma$ is overexpressed in several cancers (38-40). This overexpression constitutes a beneficial setting for tumors to proliferate and become metastatic. In fact, REG $\gamma$ was recently shown to be involved in the degradation of some regulatory proteins. For example, the cyclin-dependent kinase inhibitor p21 is specifically degraded in an ATP- and ubiquitin-independent process via the REG $\gamma$-proteasomal pathway (18). Furthermore, two other cyclin-dependent kinases inhibitors, p16 and p14, are reportedly degraded via the same pathway (17). Even more importantly, REG $\gamma$ has been shown to be involved in the MDM2-mediated p53 degradation process (20). These findings demonstrate the important role of REG $\gamma$ in tumorigenicity by regulating cell proliferation and apoptosis. In this study, we demonstrate a new role for REG $\gamma$ that is beneficial for cancer growth and development. Indeed, REG $\gamma$ overexpression not only encourages cancer progression but, as confirmed by our data, is involved in reducing the amount of polypeptides that can serve as MHC-I epitopes, thus causing an inefficient immune response against tumor cell lines and finally resulting in the invisibility of cancer cells to the host immune system.

The $\mathrm{REG} \alpha / \beta$ complex is largely located in the cytoplasm, while REG $\gamma$ is mostly a nuclear proteasomal regulator (15). REG $\gamma$ has been shown to be associated with the $20 \mathrm{~S}$ proteasome in nuclear speckles (21). Moreover, many reports have demonstrated that the $20 \mathrm{~S}$ proteasome and immunoproteasome are present in the nucleus and, more specifically, in clastosome structures (50). Considering that PTPs are produced by a nuclear translation event, it is clearly more beneficial for cells to process different PTPs in the same compartment where they are produced. In terms of the cancer hiddenness to the host immune system, it is also more advantageous for cancer cells to rapidly destroy tumor-associated PTPs (TA-PTPs) where they are produced rather than to give them the 
chance to be properly processed in the cytoplasm in a way that might generate class I epitopes and thus induce specific cancer immune responses. Therefore, by overexpressing REG $\gamma$ in the nucleus where PTPs are located, cancer cells add another layer of regulation to the immune response. Indeed, we showed that by manipulating the localization of REG $\gamma$, via overexpression of the $\Delta$ NLS variant of the regulator, we were able to restore a proper immune response against TA-PTPs. This identification of a specific site for TA-PTP degradation reinforced our recent hypothesis that, under normal conditions, MHC-I antigen epitopes are produced and processed in the nucleus, but under abnormal conditions such as cancer, TA-PTPs are still produced in the nucleus but are immediately processed by the REG $\gamma$-proteasomal complex in a specific way that minimizes the generation of MHC-I epitopes instead of be properly processed by different types of proteasomes such as the standard $20 \mathrm{~S}$ proteasome, the immunoproteasome or a REG $\alpha / \beta$-proteasomal complex, all of which were shown to be able to generate MHC-I epitopes (13,51-54)

The eukaryotic proteasome possesses three different peptidase activities. The $\beta 1$ subunit exhibits caspase-like or peptidyl-glutamyl peptide hydrolyzing (PGPH) activity, the $\beta 2$ subunit exhibits trypsin-like activity, and the $\beta 5$ subunit exhibits chymotrypsin-like activity (55). When cells are treated with interferon gamma (IFN $\gamma$ ), the expression of three additional catalytic $\beta$-subunits is stimulated. All three active constitutive $\beta$-subunits are thus replaced, in newly assembled complexes, by a corresponding IFN $\gamma$ induced $\beta$-subunits, specifically LMP2 ( $\beta 1 \mathrm{i})$, LMP7 ( $\beta 5 \mathrm{i})$ and LMP10 ( $\beta 2 \mathrm{i})$ (Mecl1), producing the so-called immunoproteasome (28). Although the heptameric REG $\alpha / \beta$ has been reported to stimulate all three proteasomal activities (12), the effect of REG $\gamma$ on proteasomal cleaving specificities appear more complex and strictly related to the exact sequence of the fluorogenic short peptides used to assess them (27). The heptameric REG $\alpha / \beta$ regulator binds to $20 \mathrm{~S}$ proteasome and immunoproteasome and in this way enhances generation of bind some, but not all, MHC-I epitopes. Two different explanations might be proposed to explain the ability of REG $\gamma$ to decrease PTPdependent antigen presentation: REG $\gamma$ might (1) inhibit PTP processing via the proteasome complex or (2) enhance the destruction by $20 \mathrm{~S}$ proteasome of MHC-I epitopes embedded in PTPs. Here, we propose that by binding to the nuclear $20 \mathrm{~S}$ proteasome REG $\gamma$ stimulates the degradation, rather than 
the proper processing, of MHC-I antigenic peptides by changing the peptidase activities of the $20 \mathrm{~S}$ core particle. In vitro degradation assays, in fact, clearly showed that several polypeptides (ranging in length between 45 and 53 residues), with size and sequences corresponding to different PTPs, are efficiently hydrolyzed by REG $\gamma$-20S proteasomes at rates only slightly lower than those obtained with $\mathrm{REGa} / \mathrm{b}-20 \mathrm{~S}$ particles. These experiments, therefore, rule out the possibility that REG $\gamma$, by binding to 20 S proteasome, may simply act as general inhibitor of proteasomal degradation of PTPs. In line with the alternative hypothesis that REG $\gamma$ may induce destruction, rather than proper processing, of specific antigenic peptides, MS analysis demonstrated that 20S proteasome is able to efficiently process and release the immunodominant epitope SIINFEKL embedded in the precursor peptide KH-52, corresponding to the sequence of the $\beta$-globin intron region. Crucially, however, when the precursor peptide KH-52 was degraded by the $20 \mathrm{~S}$ proteasome activated by REG $\gamma$, the generation of the immunodominant epitope SIINFEKL was completely abolished. Although our MS semiquantitative analysis precluded the recognition of small peptides generated following the fragmentation of SIINFEKL, the stimulation of proteasomal peptidase activities, resulting in the destruction of the antigenic peptide, appeared to be the more likely explanation for the inability to detect the immunodominant epitope in the presence of REG $\gamma$.

We recently reported that PTPs are a major source of MHC-I antigenic peptides for the endogenous and exogenous pathways. In fact, by inhibiting PTP degradation by the proteasome in tumor cell lines, we were able to increase the amounts of unprocessed PTPs entering the exogenous pathway, resulting in an increase in the activation of naïve CD8+ T cells $(7,8)$. The role we unveiled of REG $\gamma$ in the degradation of PTPs and specifically its ability to inhibit the endogenous MHC-I antigen presentation from TA-PTPs in tumors, leads to the hypothesis that this mechanism might also affect PTP-dependent exogenous MHC-I presentation. Indeed, by overexpressing REG $\gamma$ in a CRISPR REG $\gamma$ knock-out cell line, we showed that this cell line was no longer able to induce the proliferation of CD8+ T cells via the exogenous pathway. Moreover, mutated and $\triangle \mathrm{NLS}-\mathrm{REG} \gamma$ constructs played a less active role in PTP degradation, indicating that the main function of REG $\gamma$ was to modulate the activity of the nuclear proteasomal complex, with strong inhibitory consequences not only on MHC-I 
endogenous antigen presentation but also on MHC-I exogenous pathway. Thus, we are driven to speculate that cancer cells might induce the overexpression of REG $\gamma$ for both purposes: not only to inhibit MHC-I endogenous pathway but also to ensure that TA-PTPs have no chance to enter the MHC-I exogenous pathway and so to induce the proliferation of naïve CD8+ T cells.

In conclusion, the results of the present study, together with those of previous studies investigating REG $\gamma$ functions, support the idea that tumor cell lines have an established system to positively regulate REG $\gamma$ expression for specific purposes, such as to i) control cell cycle arrest, ii) control apoptosis, iii) control cell proliferation, and most importantly iv) inhibit endogenous and exogenous MHC-I antigen presentation by specifically degrading TA-PTPs encode MHC-I epitopes in order to become invisible to the host immune system.

\section{Acknowledgments:}

We thank Xiaotao Li (Department of Biochemistry and Molecular Biology, Institute of Biomedical Sciences, Shanghai, China) for the REG $\gamma$ expression plasmids. The A375 and A549 cell lines were a gift from Dr. Fabrice André, Gustave Roussy Institute. The WM3526 and WM3682 cell lines were a gift from Levi Garraway. The HT29 and T84 colon cell lines were a gift from Dr. Fanny Jaulin from the Gustave Roussy Institute. We also thank Prof. Laurence Zitvogel for valuable comments on the manuscript. This work was funded by The Avenir program (INSERM), La Fondation ARC, and Fondation Gustave Roussy. BM is supported by the Fondation Gustave Roussy, and PA is supported by the Université Paris Sud.

\section{References}

1. Joffre OP, Segura E, Savina A, Amigorena S. Cross-presentation by dendritic cells. Nature reviews Immunology 2012;12:557-69

2. Yewdell JW, Anton LC, Bennink JR. Defective ribosomal products (DRiPs): a major source of antigenic peptides for MHC class I molecules? J Immunol 1996;157:1823-6

3. Coulie PG, Lehmann F, Lethe B, Herman J, Lurquin C, Andrawiss M, et al. A mutated intron sequence codes for an antigenic peptide recognized by cytolytic $T$ lymphocytes on a human melanoma. Proceedings of the National Academy of Sciences of the United States of America 1995;92:7976-80

4. Guilloux Y, Lucas S, Brichard VG, Van Pel A, Viret C, De Plaen E, et al. A peptide recognized by human cytolytic T lymphocytes on HLA-A2 melanomas is encoded by an intron sequence of 
the $\mathrm{N}$-acetylglucosaminyltransferase $\mathrm{V}$ gene. The Journal of experimental medicine 1996;183:1173-83

5. Shastri N, Cardinaud S, Schwab SR, Serwold T, Kunisawa J. All the peptides that fit: the beginning, the middle, and the end of the MHC class I antigen-processing pathway. Immunological reviews 2005;207:31-41

6. Apcher S, Daskalogianni C, Lejeune F, Manoury B, Imhoos G, Heslop L, et al. Major source of antigenic peptides for the MHC class I pathway is produced during the pioneer round of mRNA translation. Proceedings of the National Academy of Sciences of the United States of America 2011;108:11572-7

7. Apcher S, Millot G, Daskalogianni C, Scherl A, Manoury B, Fahraeus R. Translation of prespliced RNAs in the nuclear compartment generates peptides for the MHC class I pathway. Proceedings of the National Academy of Sciences of the United States of America 2013;110:17951-6

8. Duvallet E, Boulpicante M, Yamazaki T, Daskalogianni C, Prado Martins R, Baconnais S, et al. Exosome-driven transfer of tumor-associated Pioneer Translation Products (TA-PTPs) for the MHC class I cross-presentation pathway. Oncoimmunology 2016;5:e1198865

9. Collins GA, Goldberg AL. The Logic of the 26S Proteasome. Cell 2017;169:792-806

10. Kish-Trier E, Hill CP. Structural biology of the proteasome. Annu Rev Biophys 2013;42:29-49

11. Leone P, Shin EC, Perosa F, Vacca A, Dammacco F, Racanelli V. MHC class I antigen processing and presenting machinery: organization, function, and defects in tumor cells. J Natl Cancer Inst 2013;105:1172-87

12. Whitby FG, Masters El, Kramer L, Knowlton JR, Yao Y, Wang CC, et al. Structural basis for the activation of $20 S$ proteasomes by 115 regulators. Nature 2000;408:115-20

13. Cascio P, Call M, Petre BM, Walz T, Goldberg AL. Properties of the hybrid form of the $26 \mathrm{~S}$ proteasome containing both $19 S$ and PA28 complexes. EMBO J 2002;21:2636-45

14. Cascio P. PA28alphabeta: the enigmatic magic ring of the proteasome? Biomolecules 2014;4:566-84

15. Soza A, Knuehl C, Groettrup M, Henklein P, Tanaka K, Kloetzel PM. Expression and subcellular localization of mouse $20 S$ proteasome activator complex PA28. FEBS letters 1997;413:27-34

16. Wojcik C, Tanaka K, Paweletz N, Naab U, Wilk S. Proteasome activator (PA28) subunits, alpha, beta and gamma (Ki antigen) in NT2 neuronal precursor cells and HeLa S3 cells. Eur J Cell Biol 1998;77:151-60

17. Chen X, Barton LF, Chi Y, Clurman BE, Roberts JM. Ubiquitin-independent degradation of cellcycle inhibitors by the REGgamma proteasome. Molecular cell 2007;26:843-52

18. Li X, Amazit L, Long W, Lonard DM, Monaco JJ, O'Malley BW. Ubiquitin- and ATP-independent proteolytic turnover of p21 by the REGgamma-proteasome pathway. Molecular cell 2007;26:831-42

19. Li X, Lonard DM, Jung SY, Malovannaya A, Feng Q, Qin J, et al. The SRC-3/AIB1 coactivator is degraded in a ubiquitin- and ATP-independent manner by the REGgamma proteasome. Cell 2006;124:381-92

20. Zhang Z, Zhang R. Proteasome activator PA28 gamma regulates $p 53$ by enhancing its MDM2mediated degradation. The EMBO journal 2008;27:852-64

21. Baldin V, Militello M, Thomas $Y$, Doucet C, Fic W, Boireau S, et al. A novel role for PA28gamma-proteasome in nuclear speckle organization and SR protein trafficking. Molecular biology of the cell 2008;19:1706-16

22. Cioce M, Boulon S, Matera AG, Lamond AI. UV-induced fragmentation of Cajal bodies. The Journal of cell biology 2006;175:401-13

23. Zannini L, Buscemi G, Fontanella E, Lisanti S, Delia D. REGgamma/PA28gamma proteasome activator interacts with PML and Chk2 and affects PML nuclear bodies number. Cell Cycle 2009;8:2399-407

24. Fort P, Kajava AV, Delsuc F, Coux O. Evolution of proteasome regulators in eukaryotes. Genome Biol Evol 2015;7:1363-79 
25. Boulon S, Westman BJ, Hutten S, Boisvert FM, Lamond Al. The nucleolus under stress. Molecular cell 2010;40:216-27

26. Welk V, Coux O, Kleene V, Abeza C, Trumbach D, Eickelberg O, et al. Inhibition of Proteasome Activity Induces Formation of Alternative Proteasome Complexes. The Journal of biological chemistry 2016;291:13147-59

27. Jonik-Nowak B, Menneteau T, Fesquet D, Baldin V, Bonne-Andrea C, Mechali F, et al. PIP30/FAM192A is a novel regulator of the nuclear proteasome activator PA28gamma. Proceedings of the National Academy of Sciences of the United States of America 2018;115:E6477-E86

28. Groettrup M, Kirk CJ, Basler M. Proteasomes in immune cells: more than peptide producers? Nature reviews Immunology 2010;10:73-8

29. Barton LF, Runnels HA, Schell TD, Cho Y, Gibbons R, Tevethia SS, et al. Immune defects in 28kDa proteasome activator gamma-deficient mice. J Immunol 2004;172:3948-54

30. Murata S, Kawahara H, Tohma S, Yamamoto K, Kasahara M, Nabeshima Y, et al. Growth retardation in mice lacking the proteasome activator PA28gamma. The Journal of biological chemistry 1999;274:38211-5

31. Shastri N, Gonzalez F. Endogenous generation and presentation of the ovalbumin peptide/Kb complex to T cells. J Immunol 1993;150:2724-36

32. Perchellet A, Stromnes I, Pang JM, Goverman J. CD8+ T cells maintain tolerance to myelin basic protein by 'epitope theft'. Nat Immunol 2004;5:606-14

33. Cascio P, Goldberg AL. Preparation of hybrid (19S-20S-PA28) proteasome complexes and analysis of peptides generated during protein degradation. Methods in enzymology 2005;398:336-52

34. Raule M, Cerruti F, Benaroudj N, Migotti R, Kikuchi J, Bachi A, et al. PA28alphabeta reduces size and increases hydrophilicity of $20 \mathrm{~S}$ immunoproteasome peptide products. Chem Biol 2014;21:470-80

35. Raule M, Cerruti F, Cascio P. Enhanced rate of degradation of basic proteins by $26 \mathrm{~S}$ immunoproteasomes. Biochim Biophys Acta 2014;1843:1942-7

36. Rappsilber J, Ishihama $Y$, Mann M. Stop and go extraction tips for matrix-assisted laser desorption/ionization, nanoelectrospray, and LC/MS sample pretreatment in proteomics. Anal Chem 2003;75:663-70

37. Rappsilber J, Mann M, Ishihama Y. Protocol for micro-purification, enrichment, prefractionation and storage of peptides for proteomics using StageTips. Nat Protoc 2007;2:1896-906

38. Wang X, Tu S, Tan J, Tian T, Ran L, Rodier JF, et al. REG gamma: a potential marker in breast cancer and effect on cell cycle and proliferation of breast cancer cell. Medical oncology 2011;28:31-41

39. Chai F, Liang Y, Bi J, Chen L, Zhang F, Cui Y, et al. High expression of REGgamma is associated with metastasis and poor prognosis of patients with breast cancer. Int J Clin Exp Pathol 2014;7:7834-43

40. He J, Cui L, Zeng Y, Wang G, Zhou P, Yang $Y$, et al. REGgamma is associated with multiple oncogenic pathways in human cancers. BMC Cancer 2012;12:75

41. Giles KM, Brown RA, Ganda C, Podgorny MJ, Candy PA, Wintle LC, et al. microRNA-7-5p inhibits melanoma cell proliferation and metastasis by suppressing RelA/NF-kappaB. Oncotarget 2016;7:31663-80

42. Xiong $S$, Zheng $\mathrm{Y}$, Jiang $\mathrm{P}$, Liu R, Liu $\mathrm{X}$, Chu Y. MicroRNA-7 inhibits the growth of human nonsmall cell lung cancer A549 cells through targeting BCL-2. Int J Biol Sci 2011;7:805-14

43. Kefas B, Godlewski J, Comeau L, Li Y, Abounader R, Hawkinson M, et al. microRNA-7 inhibits the epidermal growth factor receptor and the Akt pathway and is down-regulated in glioblastoma. Cancer Res 2008;68:3566-72 
44. Saydam O, Senol O, Wurdinger T, Mizrak A, Ozdener GB, Stemmer-Rachamimov AO, et al. miRNA-7 attenuation in Schwannoma tumors stimulates growth by upregulating three oncogenic signaling pathways. Cancer Res 2011;71:852-61

45. Xiong $S$, Zheng $Y$, Jiang $P$, Liu R, Liu X, Qian J, et al. PA28gamma emerges as a novel functional target of tumour suppressor microRNA-7 in non-small-cell lung cancer. British journal of cancer 2014;110:353-62

46. Ali A, Wang Z, Fu J, Ji L, Liu J, Li L, et al. Differential regulation of the REGgamma-proteasome pathway by $\mathrm{p} 53 /$ TGF-beta signalling and mutant p53 in cancer cells. Nature communications 2013;4:2667

47. Kanai K, Aramata S, Katakami S, Yasuda K, Kataoka K. Proteasome activator PA28gamma stimulates degradation of GSK3-phosphorylated insulin transcription activator MAFA. J Mol Endocrinol 2011;47:119-27

48. Kisselev AF, Akopian TN, Woo KM, Goldberg AL. The sizes of peptides generated from protein by mammalian 26 and $20 \mathrm{~S}$ proteasomes. Implications for understanding the degradative mechanism and antigen presentation. The Journal of biological chemistry 1999;274:3363-71

49. Nussbaum AK, Dick TP, Keilholz W, Schirle M, Stevanovic S, Dietz K, et al. Cleavage motifs of the yeast $20 S$ proteasome beta subunits deduced from digests of enolase 1 . Proceedings of the National Academy of Sciences of the United States of America 1998;95:12504-9

50. Lafarga M, Berciano MT, Pena E, Mayo I, Castano JG, Bohmann D, et al. Clastosome: a subtype of nuclear body enriched in 195 and $20 \mathrm{~S}$ proteasomes, ubiquitin, and protein substrates of proteasome. Molecular biology of the cell 2002;13:2771-82

51. Guillaume B, Chapiro J, Stroobant V, Colau D, Van Holle B, Parvizi G, et al. Two abundant proteasome subtypes that uniquely process some antigens presented by HLA class I molecules. Proceedings of the National Academy of Sciences of the United States of America 2010;107:18599-604

52. Morel S, Levy F, Burlet-Schiltz O, Brasseur F, Probst-Kepper M, Peitrequin AL, et al. Processing of some antigens by the standard proteasome but not by the immunoproteasome results in poor presentation by dendritic cells. Immunity 2000;12:107-17

53. Van den Eynde BJ, Morel S. Differential processing of class-I-restricted epitopes by the standard proteasome and the immunoproteasome. Current opinion in immunology 2001;13:147-53

54. Vigneron N, Van den Eynde BJ. Proteasome subtypes and the processing of tumor antigens: increasing antigenic diversity. Current opinion in immunology 2012;24:84-91

55. Livneh I, Cohen-Kaplan V, Cohen-Rosenzweig C, Avni N, Ciechanover A. The life cycle of the $26 \mathrm{~S}$ proteasome: from birth, through regulation and function, and onto its death. Cell Res 2016;26:869-85

\section{Figure Legends}

Figure 1: Inverse correlation between the expression of REG $\gamma$ and antigen presentation in multiple cancer cell lines. a) REG $\gamma$ mRNA levels were analyzed by qPCR in different cell lines and normalized to $\beta$-actin mRNA levels. The MRC5 cell line was used as a reference. Experiments were performed in triplicate. Data are expressed as the mean \pm SEM from three technical replicates. b) Western blot analysis and quantification (relative to the housekeeping protein $\beta$-actin) of REG $\gamma$ expression. Protein levels are indicated below each gel. c) REG $\gamma$ protein localization was determined 
by immunofluorescence in several tumor cell lines and in the MRC5 normal cell line. REG $\square$ was stained with Alexa Fluor 488, and nuclei were counterstained with DAPI. Cells were analyzed by confocal microscopy. As expected, the regulator REG $\gamma$ was localized in the nucleus in all tested cell lines. d, e) All cell types were transfected in vitro with $\beta$-Glob-intron-SL8 (d, left panel) or $\beta$-Globintron-MBP(79-87) (d, right panel), $\beta$-Glob-exon-SL8 (e, left panel) and OVA cDNA (e, right panel) expression constructs. The cells were incubated with the SL8-specific CD8+ T cell hybridoma (B3Z) for $16 \mathrm{~h}$. The data show the average of at least three independent experiments \pm SD minus the values from mock-transfected cells. Free SL8 peptides were added to the cells to ensure that the T cell assays were performed under non-saturation conditions and that the expression of MHC-I molecules was not affected.

Figure 2: Exogenous REG $\gamma$ overexpression decreases antigen presentation. a) MRC5 and A549 were transfected with a construct expressing REG $\gamma$ WT from 0.0625 to $0.5 \mu \mathrm{g}$ or a corresponding empty construct for $48 \mathrm{~h}$. REG $\gamma$ protein levels were examined by Western blotting using $\beta$-actin as a loading control. Protein levels are indicated below each gel. b) MRC5 and A549 were transfected with a construct expressing Flag-REG $\gamma$ WT $(0.25 \mu \mathrm{g})$. The Flag tag was stained with Alexa Fluor 488, and the nuclei were stained with DAPI. Cells were analyzed by confocal microscopy. As expected, exogenous transfected REG $\gamma$ WT localized in the nuclei. c) MRC5 cells (left panel) and A549 cells (right panel) were transfected with the Glob-intron-SL8 construct $(0.5 \mu \mathrm{g})$. The cells were incubated with the SL8-specific CD8+ T cell hybridoma (B3Z) for $16 \mathrm{~h}$. The data show the average of at least three independent experiments \pm SD minus the values from mock-transfected cells. d) MRC5 cells were transfected with a REG $\gamma$ WT construct for $48 \mathrm{~h}$. The increase in REG $\gamma$ protein expression had no effect on the expression of REG $\alpha$ protein. e) MRC5 cells were transfected with increasing amounts (from 0 to $0.5 \mu \mathrm{g}$ ) of a Glob-intron-SL8 construct for $48 \mathrm{~h}$. At $36 \mathrm{~h}$ post-transfection, the cells were then treated overnight with epoxomicin $(300 \mathrm{nM})$. Next, the cells were incubated with the B3Z T cell hybridoma for $16 \mathrm{~h}$. The data show the average of at least three independent experiments \pm SD. $* * * \mathrm{p}$ $<0.001, * \mathrm{p}<0.05$ (unpaired t-test). f) Western blot analysis and quantification (relative to the 
housekeeping protein $\beta$-actin) of p21 expression in MRC5 cells treated overnight with epoxomicin at $300 \mathrm{nM}$. Protein levels are indicated below each gel. As expected, p21 protein levels increased after 12 $\mathrm{h}$ of epoxomicin treatment, even though the treated cells overexpressed the exogenous regulator REG $\gamma$.

Figure 3: Knockdown and knock-out of the expression of the regulator REG $\gamma$ promotes antigen presentation. The A375, WM3526, WM3682, HT29 and T84 cell lines were transfected with siRNA specific for REG $\gamma$ (50 and $100 \mathrm{nM}$ ) or siRNA negative control (0) for $48 \mathrm{~h}$ and then analyzed by RTqPCR (a), Western blotting (b) and a T cell assay (c). a) Experiments were performed in triplicate. Data are expressed as the mean \pm SEM of three technical replicates. b) Western blotting was performed to analyze and quantify REG $\gamma$ and $\mathrm{p} 21$ expression, and $\beta$-actin was used as a reference protein. The relative protein level is indicated below each gel. c) Cell lines were transfected with the Glob-intron-SL8 construct $(0.5 \mu \mathrm{g})$ and treated with different concentrations of siRNA specific for $\operatorname{REG} \gamma(0,50$ and $100 \mathrm{nM})$. After $48 \mathrm{~h}$, the different cell lines were incubated with the B3Z T cell hybridoma for $16 \mathrm{~h}$. The data show the average of at least three independent experiments \pm SD minus the values from mock-transfected cells. d) qPCR analysis of A375 cells transfected with siRNA specific for REG $\gamma(50$ and $100 \mathrm{nM}$ ) was performed. The inhibition of REG $\gamma$ mRNA levels was quantified and normalized to $\beta$-actin mRNA levels. Experiments were performed in triplicate. Data are expressed as the mean \pm SEM from three technical replicates. e) Western blot analysis and quantification (relative to the housekeeping protein $\beta$-actin) of REG $\gamma$ and $\mathrm{p} 21$ expression. Protein levels are indicated below each gel. f) The A375cXI and A375 cell lines were both transfected with the Glob-intron-SL8 construct ( $0.5 \mu \mathrm{g})$ for $48 \mathrm{~h}$, and REG $\gamma$-siRNA (50 or $100 \mathrm{nM}$ ) was only added to A375 cells. The cells were incubated with the B3Z T cell hybridoma for $16 \mathrm{~h}$. The data show the average of at least three independent experiments \pm SD minus the values from mock-transfected cells. $* * * \mathrm{p}<0.001, * * \mathrm{p}<0.01, * \mathrm{p}<0.05$ (unpaired t-test). g) A375 and A375cXI cells were transfected with the Glob-intron-SL8 construct vector for $48 \mathrm{~h}$. The transfection efficacy was analyzed by FACS (top panel). Human tumor cell lines were transfected with a construct expressing the $\mathrm{H}-2 \mathrm{~K}^{\mathrm{b}}$ gene and 
stimulated with extracellular SIINFEKL synthetic peptide for $15 \mathrm{~min}$. The transfection efficacy of the MHC class I kb molecules was analyzed by FACS (bottom panels). Controls were stained with mouse IgG1 K isotype control APC. Data are presented as percentages.

Figure 4: REG $\gamma$ regulates the nuclear proteasomal pathway. a) Western blotting was performed to analyze and quantify REG $\gamma$, REG $\alpha$ and p21 protein expression in A375cXI cells (relative to the housekeeping protein $\beta$-actin). The cells were transfected with a construct expressing REG $\gamma$ WT or the mutated REG $\gamma \mathrm{N} 151 \mathrm{Y}$ at different concentrations or with a corresponding empty construct for $48 \mathrm{~h}$. Protein levels are indicated below each gel. b) Cells were transfected with the Glob-intron-SL8 construct $(0.5 \mu \mathrm{g})$ and then were incubated with the B3Z $\mathrm{T}$ cell hybridoma for $16 \mathrm{~h}$. Data are the average of at least three independent experiments \pm SD minus the values from mock-transfected cells. c) A375cXI cells were transfected with a construct expressing a Flag-tagged REG $\gamma$ WT or a Flagtagged REG $\gamma$ N151Y. The Flag tag was stained with Alexa Fluor 488, and the nuclei were stained with DAPI. Cells were analyzed by confocal microscopy. As expected, the exogenous REG $\gamma$ WT and REG 7 N151Y proteins were localized in the nuclei. d) The interactions of the 20S proteasome with the exogenous REG $\gamma$ WT and REG $\gamma$ N151Y constructs were analyzed using DuoLink. PLA was performed using an antibody against the Flag tag and $\alpha 4$ subunit - of the 20S proteasome. Staining was analyzed by confocal microscopy. e) Immunoprecipitation was performed using the REG $\gamma$ antibody, and Western blotting was carried out using an antibody against the 20S_ $\alpha 6$ subunit. This experiment confirmed the interaction of the $20 \mathrm{~S}$ proteasome and exogenous REG $\gamma$ WT or REG $\gamma$ N151Y. f) Western blot analysis and quantification (relative to the housekeeping protein $\beta$-actin) of REG $\gamma$, REG $\gamma$ and p21 protein levels in A375c.XI cells transfected with a construct expressing REG $\gamma$ WT or REG $\gamma$ $\triangle \mathrm{NLS}$ at different concentrations or with a corresponding empty construct for $48 \mathrm{~h}$. Protein levels are indicated below each gel. g) Cells were transfected with Glob-intron-SL8 construct ( $0.5 \mu \mathrm{g})$. The cells were then incubated with the B3Z T cell hybridoma for $16 \mathrm{~h}$. The data show the average of at least three independent experiments \pm SD minus the values from mock-transfected cells. 
Figure 5: REG $\gamma$ promotes the degradation of MHC-I PTP-derived antigenic epitopes. a) A375, WM3526, WM3682, HT29, T84, A549, MRC5 and A375cXI cell lines expressing the Glob-intronSL8 construct $(0.5 \mu \mathrm{g})$ were cultured with BMDCs for $24 \mathrm{~h}$. The BMDCs were then co-cultured with the SL8-specific CD8+ T cell hybridoma (B3Z) for $16 \mathrm{~h}$, and $\mathrm{T}$ cell activation was estimated by

measuring $\beta$-galactosidase levels. The data show the average of at least three independent experiments \pm SD minus the values from mock-transfected cells. $* * * \mathrm{p}<0.001, * * \mathrm{p}<0.01, * \mathrm{p}<0.05$ (unpaired ttest). Hydrolysis rates of KH-52 and KH-53 (b) and MP-45 and MP-46 (c) peptides. Precursor substrates were incubated with human $20 \mathrm{~S}$ constitutive proteasomes (h20Sc) alone or activated by $\mathrm{REG} \gamma$ or REG $\alpha / \beta$, and the amino groups released were measured with fluorescamine at the indicated time points. Data are representative of three independent experiments. d) Heatmap comparison of the abundance of significant peptides generated from proteasomal degradation of KH-52 precursor peptide in the h20Sc (biological and technical replicates of samples designated A and C, respectively) and REG $\gamma$-h20Sc (biological and technical replicates of samples designated B and D, respectively) samples. Differences and similarities in peptide intensities (normalized to the Z-score) are shown; green indicates decreased levels, and red indicates increased levels. Data were obtained from supervised hierarchical clustering analysis by applying a t-test and a p-value of 0.05. e) Box plot of SIINFKEL peptide intensity calculated by MaxQuant in the h20Sc (in green) and REG $\gamma$-h20Sc (in blue) samples.

Figure 6: Knockout of REG $\gamma$ gene causes tumor growth defect and changes on the tumor immunopeptidome. a) The B6 fibroblast and MCA205 sarcoma cell lines were transfected with $\beta$ Glob-intron-SL8 constructs. Cells were co-cultured with the SL8-specific CD8+ T cell hybridoma (B3Z) for $16 \mathrm{~h}$. Upper panel shows the protein level of REG $\gamma$ in the tumor cells assessed by Western Blot (quantification relative to the housekeeping protein $\beta$-actin). Lower panel shows the relative level of antigen presentation in the tumor cells. b) The MCA205 sarcoma cell line and the Cas9-REG $\square$ MCA205 clones were transfected with the $\beta$-Glob-intron-SL8 construct for $48 \mathrm{~h}$. The cells were then co-cultured with the SL8-specific CD8+ T cell hybridoma (B3Z) for $16 \mathrm{~h}$. Upper panel shows the 
protein level of REG $\gamma$ in the tumor cells assessed by Western Blot (quantification relative to the housekeeping protein $\beta$-actin). Lower panel shows the relative level of antigen presentation in the tumor cells. c) Tumor size in area of sarcoma MCA205 WT and Cas9-REG $\gamma$ MCA205 clones subcutaneously inoculated into the right flank of immunocompetent C57BL/6 mice. d) Tumor size in area of sarcoma MCA205 WT and Cas9-REG $\gamma$ MCA205 clones subcutaneously inoculated into the right flank of immunodeficient $\mathrm{Nu} / \mathrm{Nu}$ mice or immunocompetent C57BL/6 mice (left panel). Relative growth of MCA205 WT and Cas9-REG $\gamma$ MCA205 clones in immunodeficient Nu/Nu mice compared to immunocompetent C57BL/6 mice. Data are given as mean \pm SEM. $* \mathrm{p}<0.05$, $* * \mathrm{p}<0.01$ (ANOVA with Tukey's multiple comparaison test comparing all groups). 
A)

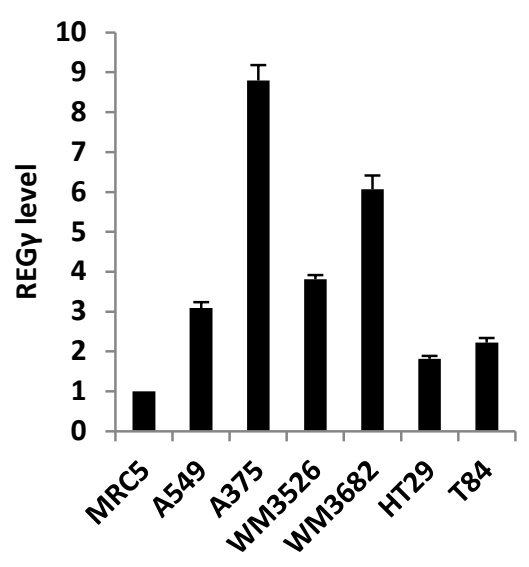

D)

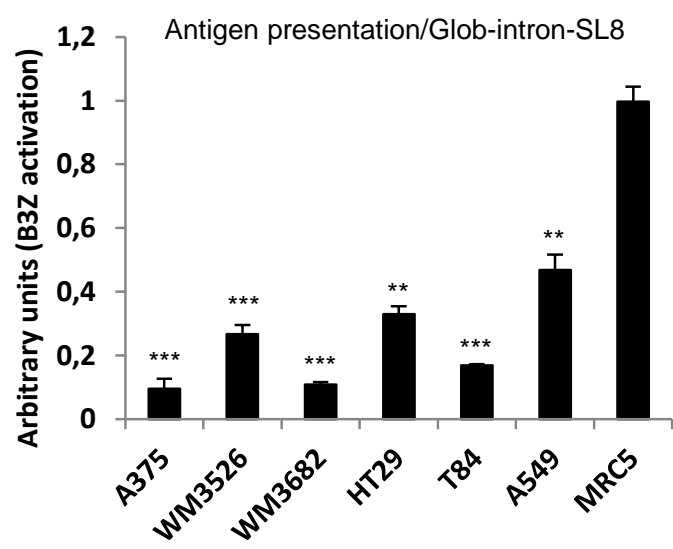

E)

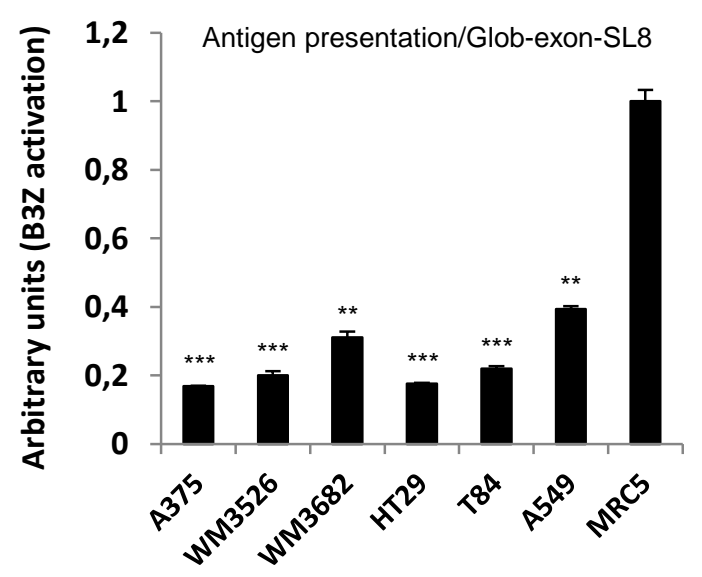

B)

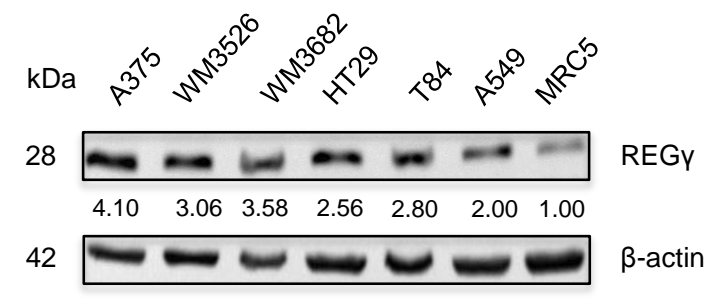

C)

A375
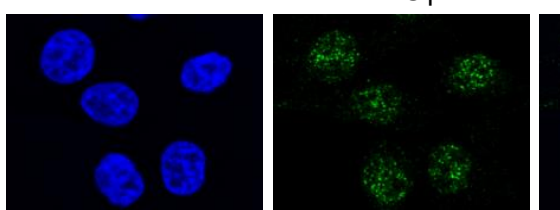

Merge

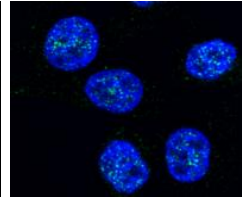

WM3526
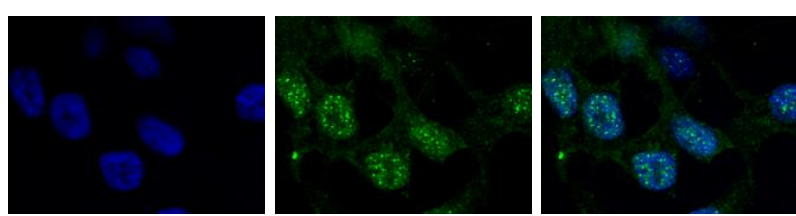

WM3682

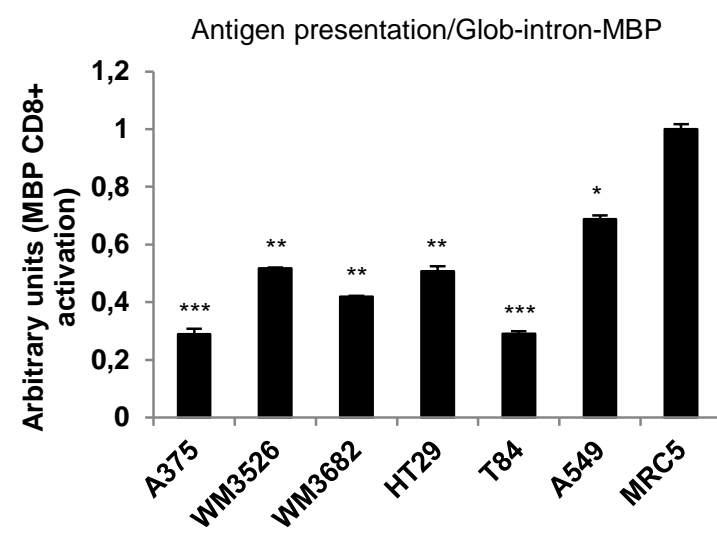

HT29
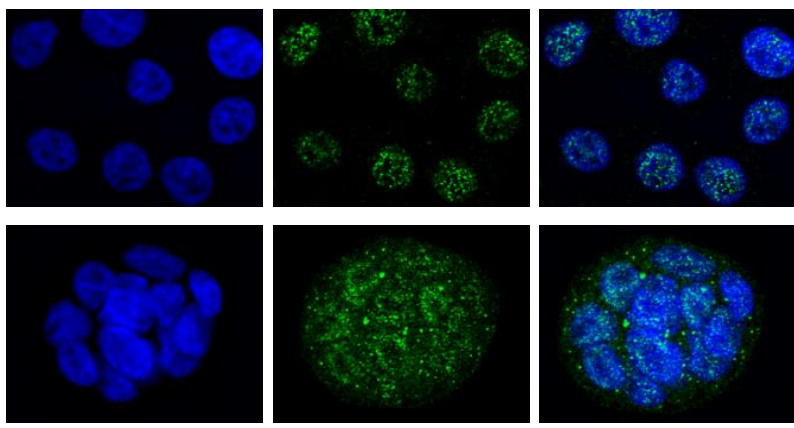

T84
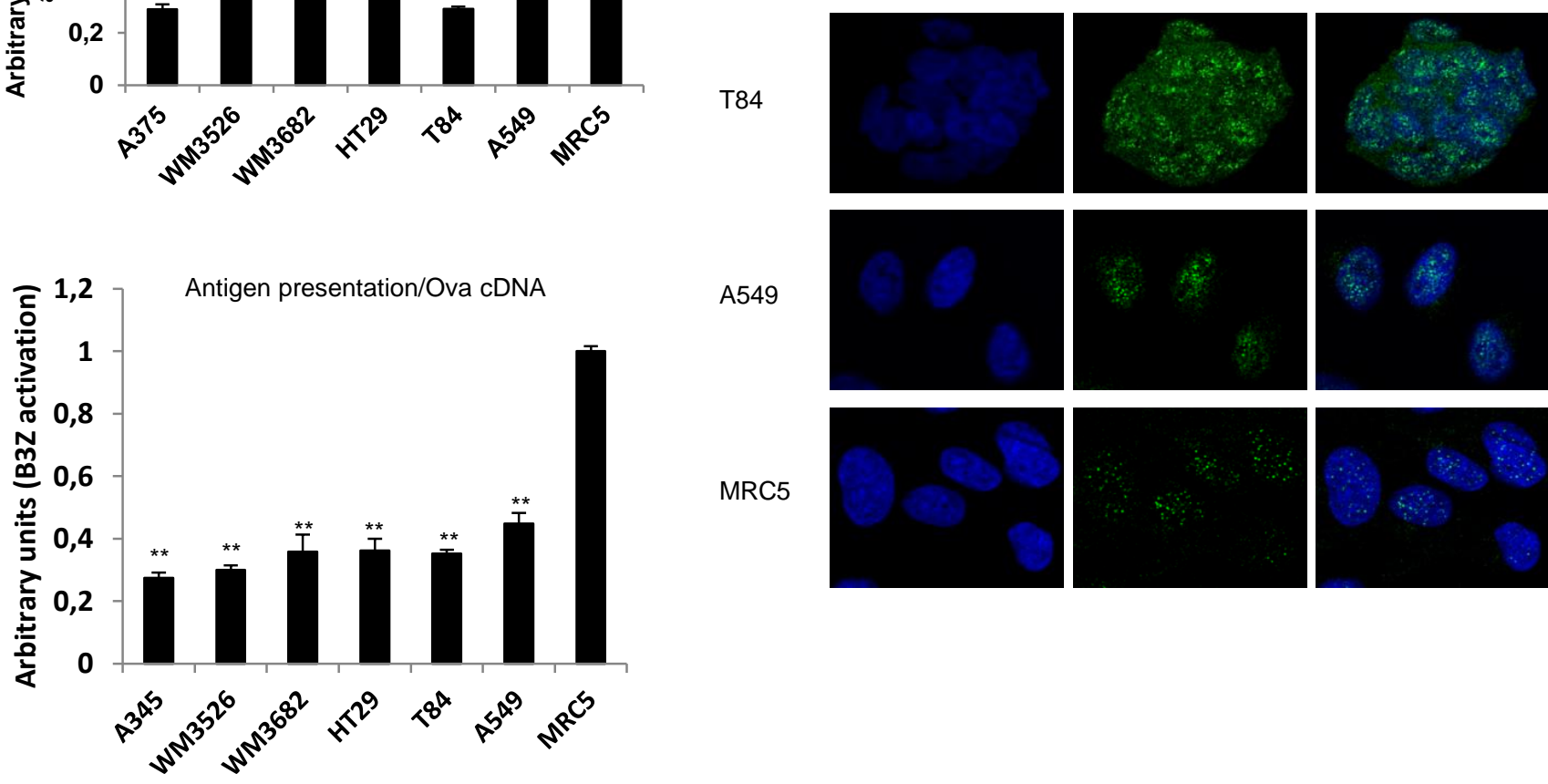

A549
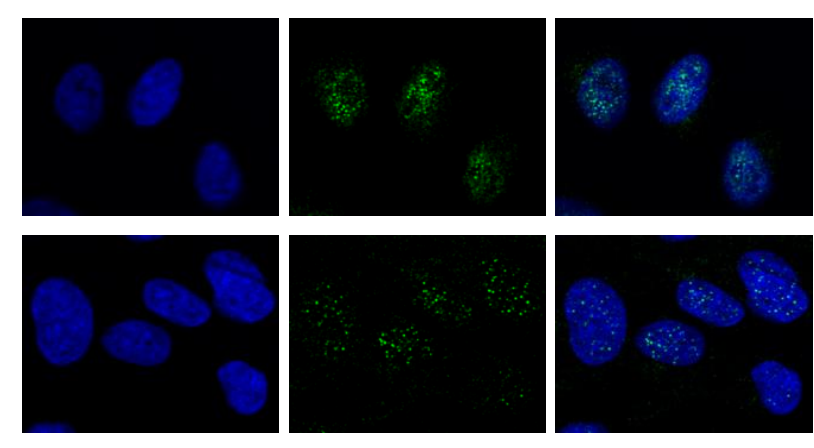
A)

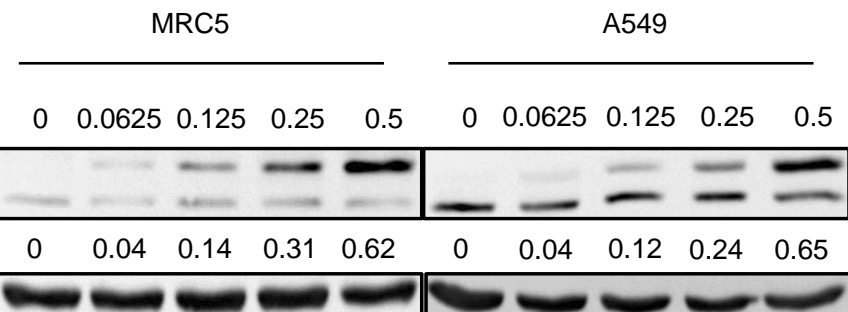

Flag-REGy WT $(\mu \mathrm{g})$ exogenous REGy endogenous REGy $\beta$-actin
B)

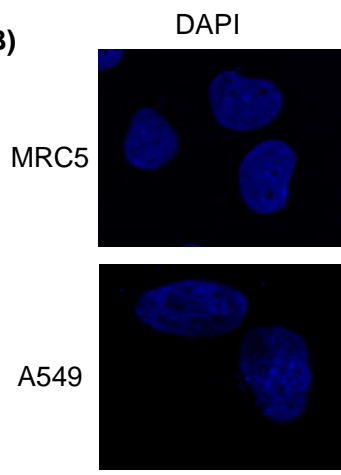

Flag-REGy

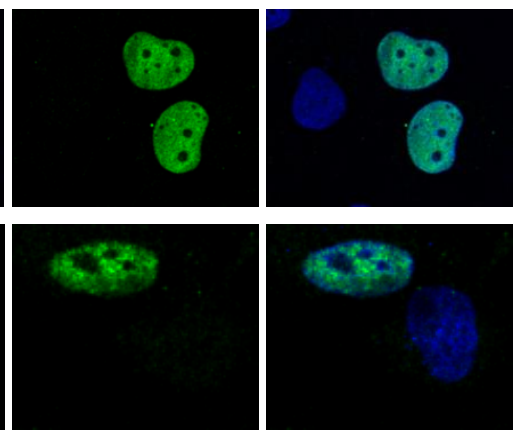

C)

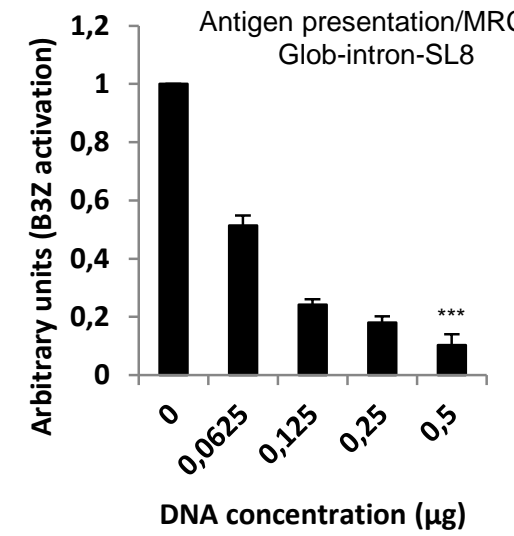

E)

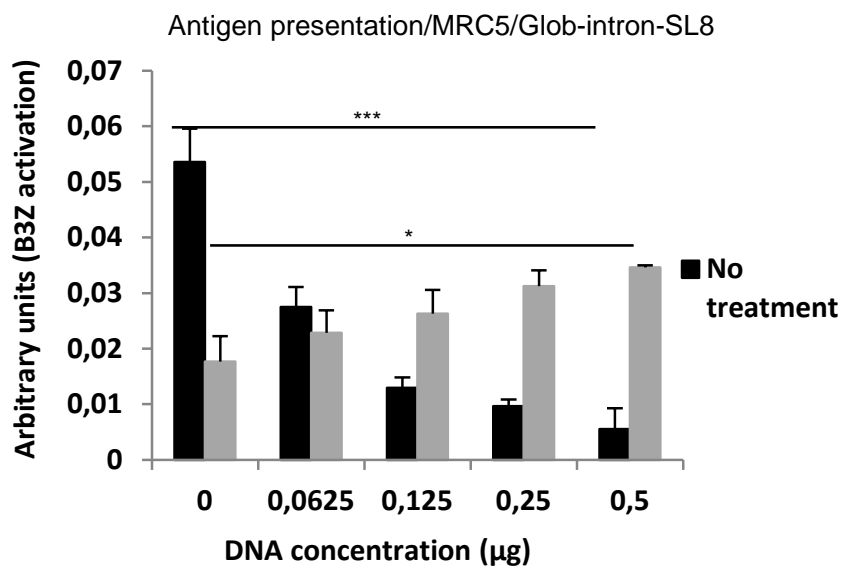

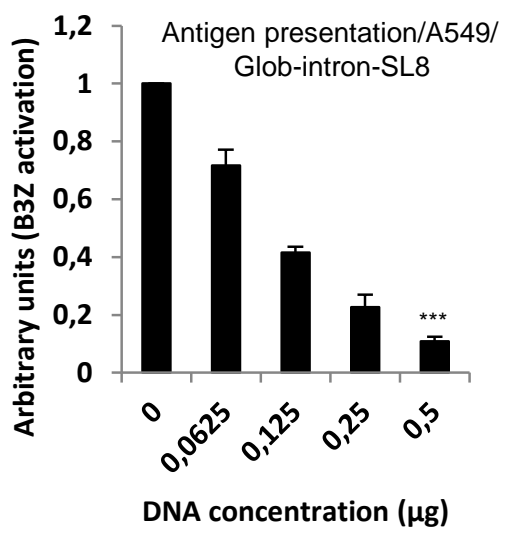

D)

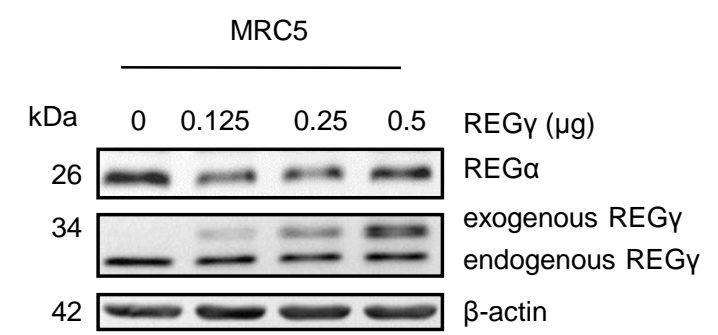

F)

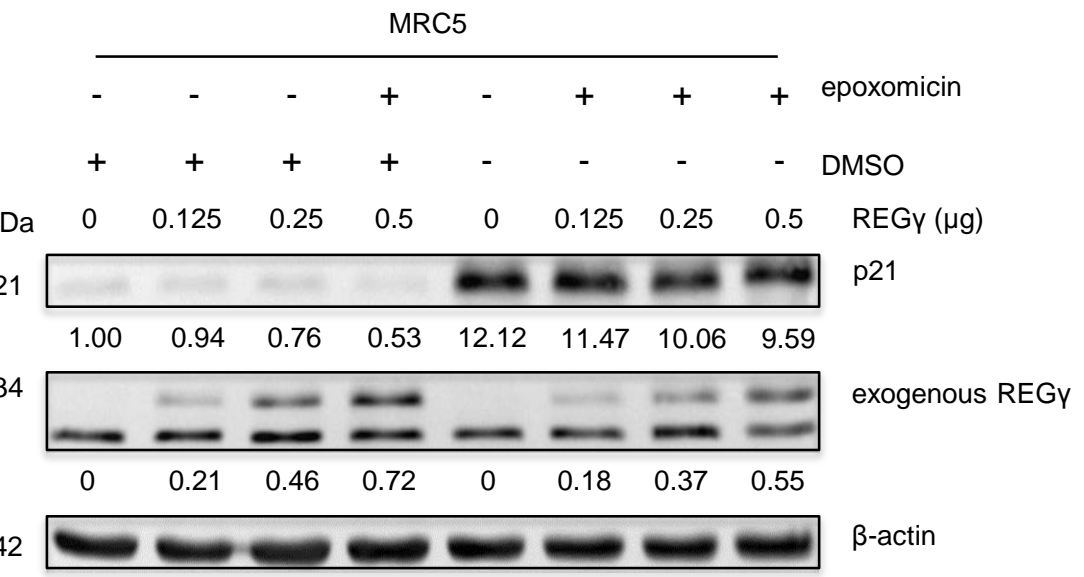


Figure 3
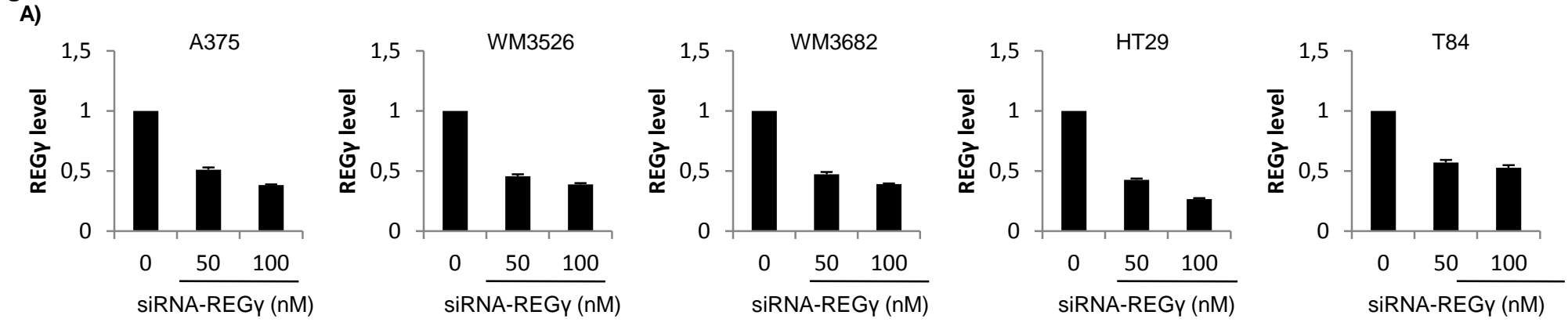

B)

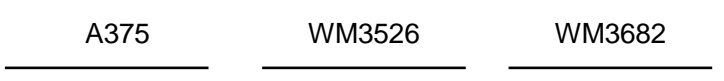

HT29

T84

$\begin{array}{lllllllllllllllll}\mathrm{kDa} & 0 & 50 & 100 & 0 & 50 & 100 & 0 & 50 & 100 & 0 & 50 & 100 & 0 & 50 & 100 & \text { siRNA-REGy (nM) }\end{array}$

21

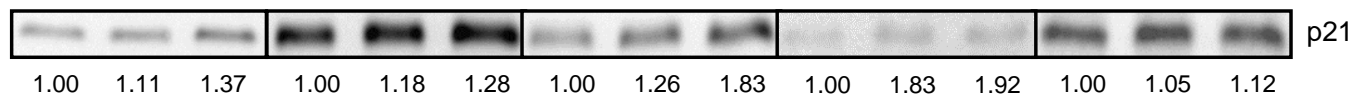

28

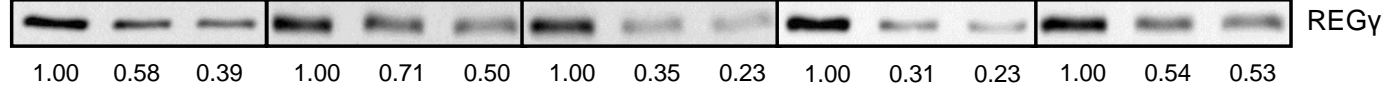

42

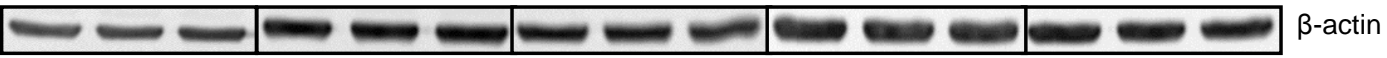

D)

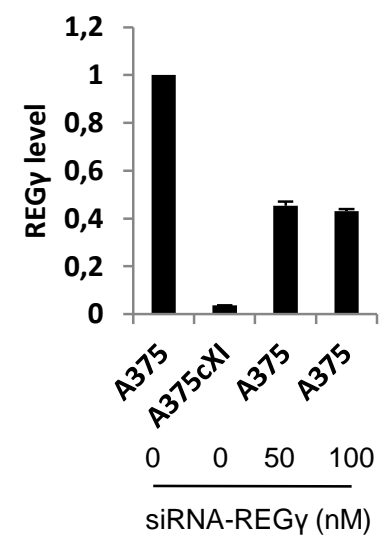

E)

$0 \quad 0 \quad 50 \quad 100$ siRNA-REGy

$\mathrm{kD}$

21

28

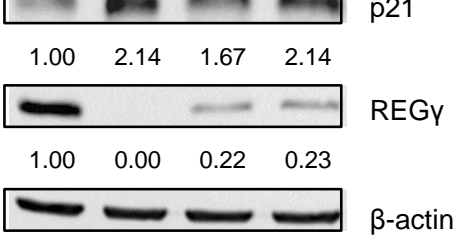

42

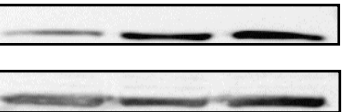

F)

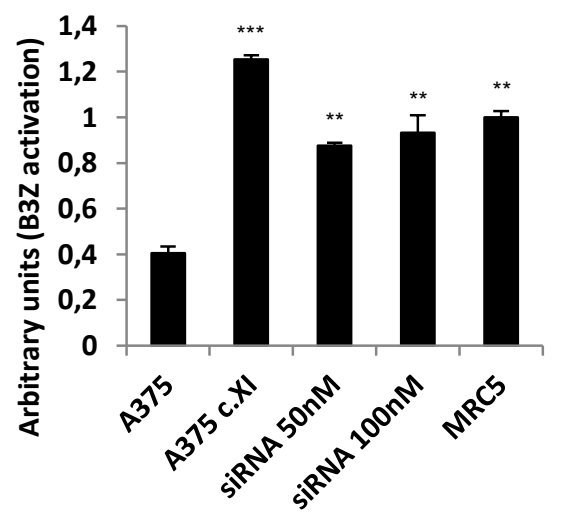

G)

C)

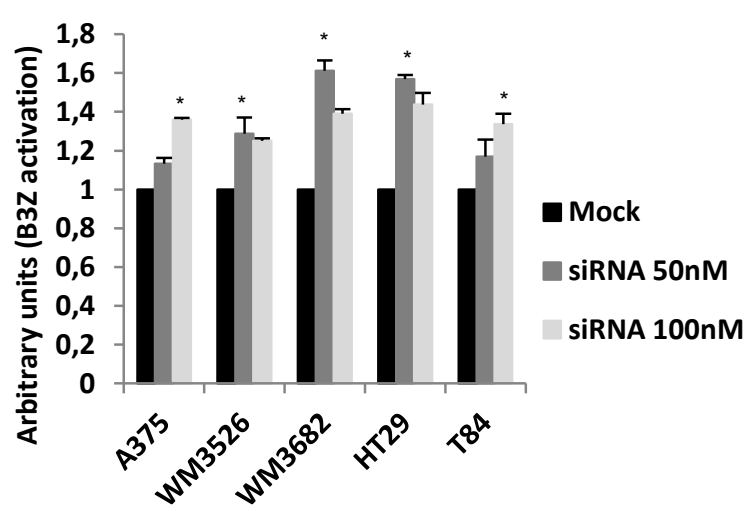

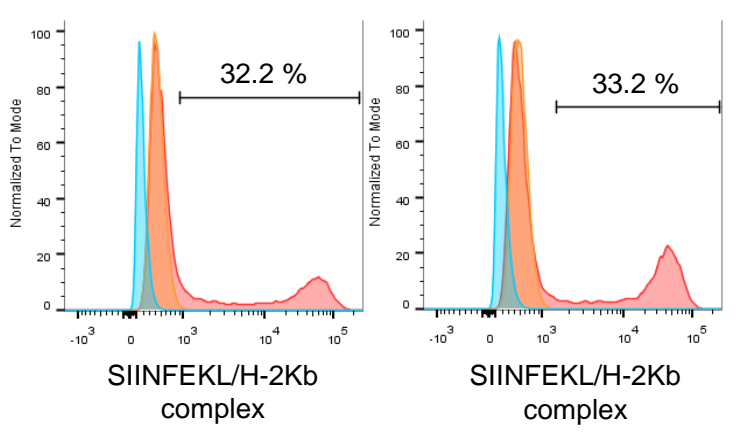




\section{Figure 5}

A)

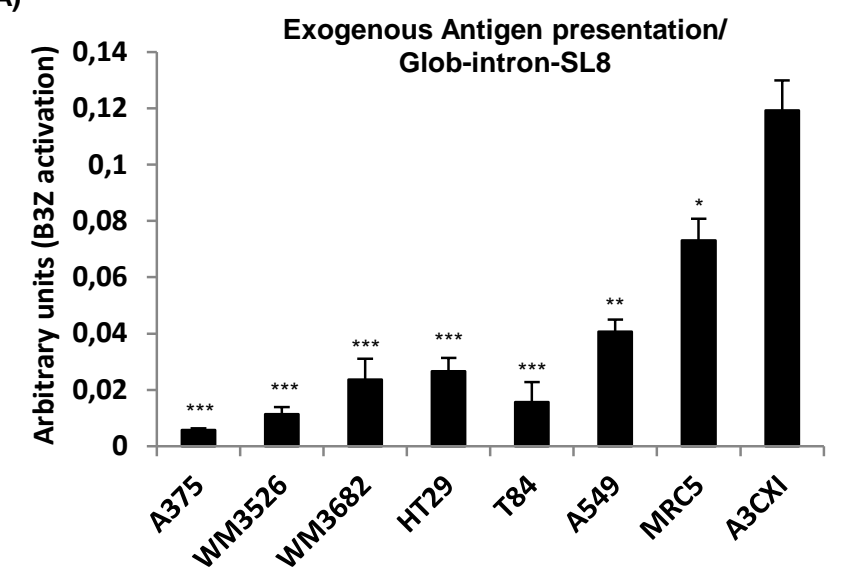

B)

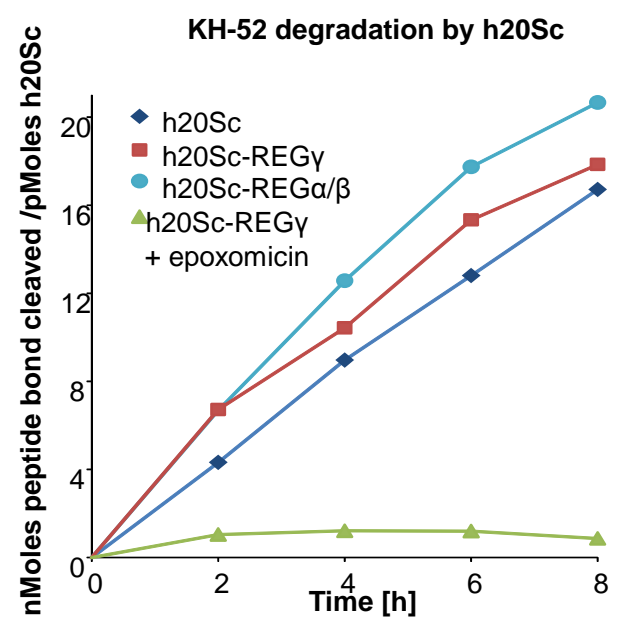

KH-53 degradation by h20Sc

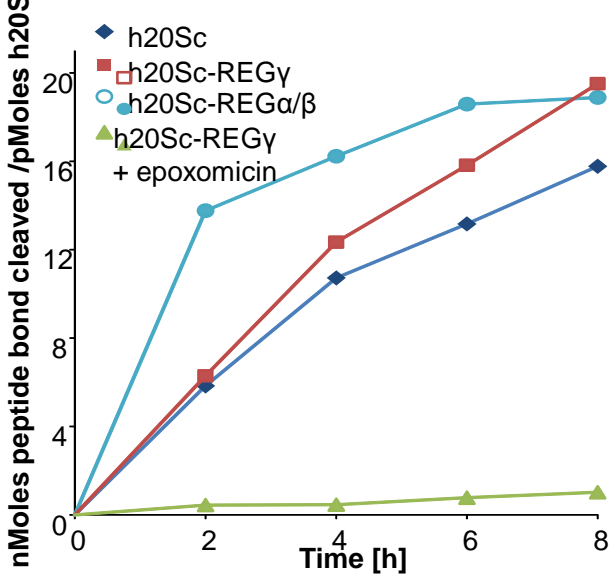

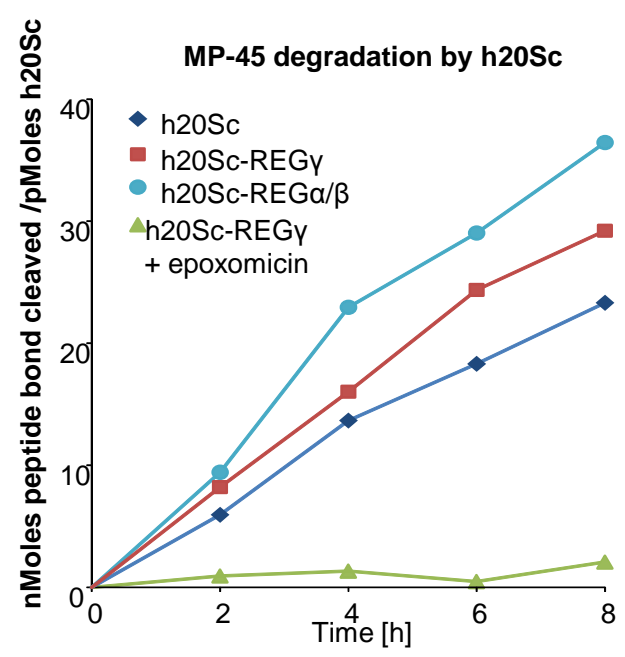

E)

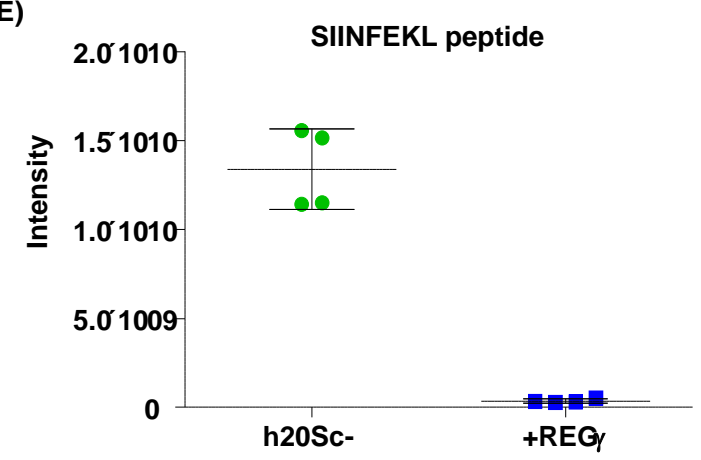

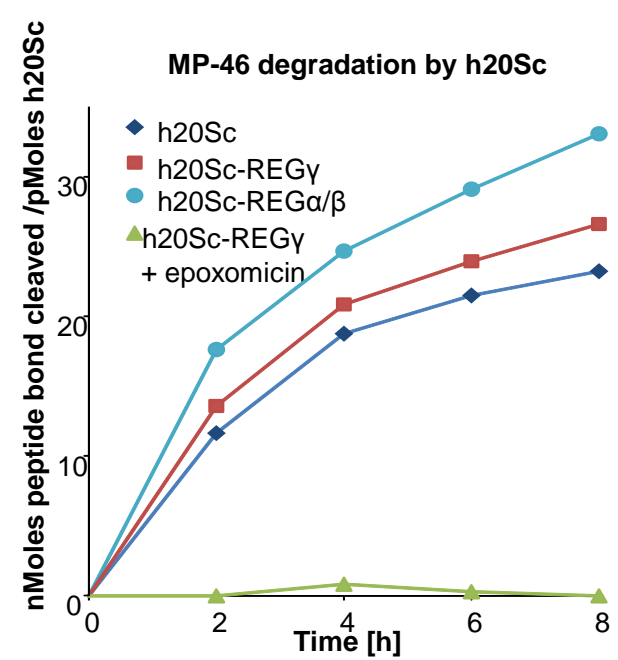

D)

HeatMap - Supervised - A C vs. B D

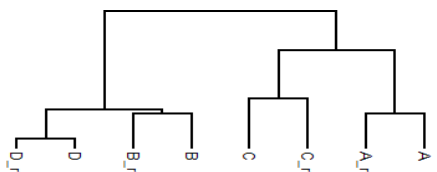

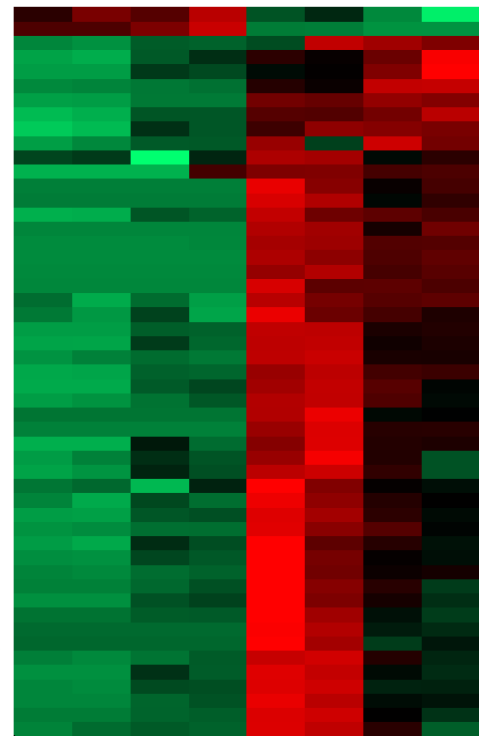


